\title{
Equivariant diagrams of spaces
}

\author{
EMANUELE DOTTO
}

\begin{abstract}
We generalize two classical homotopy theory results, the Blakers-Massey theorem and Quillen's Theorem B, to $G$-equivariant cubical diagrams of spaces, for a discrete group $G$. We show that the equivariant Freudenthal suspension theorem for permutation representations is a direct consequence of the equivariant Blakers-Massey theorem. We also apply this theorem to generalize to $G$-manifolds a result about cubes of configuration spaces from embedding calculus. Our proof of the equivariant Theorem B involves a generalization of the classical Theorem B to higher-dimensional cubes, as well as a categorical model for finite homotopy limits of classifying spaces of categories.
\end{abstract}

55P91; 55Q91

\section{Introduction}

Equivariant diagrams of spaces and their homotopy colimits have broad applications throughout topology. They are used in [12] for decomposing classifying spaces of finite groups, to study posets of $p$-groups in [19], for splitting Thom spectra in [18], and even in the definition of the cyclic structure on THH of [3]. In previous joint work with K Moi [7], the authors develop an extensive theory of equivariant diagrams in a general model category, and they study the fundamental properties of their homotopy limits and colimits. In the present paper we restrict our attention to $G$-diagrams in the category of spaces. The special feature of $G$-diagrams of spaces is the existence of generalized fixed-point functors that preserve and reflect equivalences. We study these functors and we use them to generalize the Blakers-Massey theorem and Quillen's Theorem B to equivariant cubical diagrams.

Throughout the paper, $G$ is going to be a discrete group. Let $I$ be a small category with a $G$-action. A $G$-structure on a diagram $X: I \rightarrow$ Top is a sort of generalized $G$-action on $X$ that depends on the way $G$ acts on $I$; see Definition 1.1. The key feature of a $G$-structure is that it induces a $G$-action on the homotopy limit and on the homotopy colimit of $X$, as we discuss in Section 1.1. These equivariant constructions can be described as derived functors in a suitable model categorical context; see [7]. In the present paper, we will focus mostly on $G$-diagrams of cubical shape. If $J$ is 
a finite $G$-set, the poset category $I=\mathcal{P}(J)$ of subsets of $J$ ordered by inclusion inherits a $G$-action. A $J$-cube of spaces is a diagram $X: \mathcal{P}(J) \rightarrow$ Top equipped with a $G$-structure. The canonical maps

$$
\phi: X_{\varnothing} \longrightarrow \underset{\mathcal{P}_{0}(J)}{\operatorname{holim}} X, \quad \psi: \underset{\mathcal{P}_{1}(J)}{\operatorname{hocolim}} X \longrightarrow X_{J}
$$

are $G$-equivariant, where $\mathcal{P}_{0}(J)$ and $\mathcal{P}_{1}(J)$ are the category $\mathcal{P}(J)$ respectively with the initial and the final object removed. Given a function $\nu:\{H \leq G\} \rightarrow \mathbb{N}$ which is invariant on conjugacy classes, we say that $X$ is $v$-cartesian if the restriction of $\phi$ on $H$-fixed points is $v(H)$-connected. Dually, $X$ is $v$-cocartesian if $\psi$ is $v(H)-$ connected on $H$-fixed points. In Section 2.1, we prove a formula that describes how cartesian a $J$-cube $X$ is, depending on how cocartesian its subcubes are. The precise formula is technical, but it is roughly of the following form.

Theorem (Equivariant Blakers-Massey) A $J$-cube of spaces $X: \mathcal{P}(J) \rightarrow$ Top is $v$-cartesian, where $v:\{H \leq G\} \rightarrow \mathbb{N}$ is a function of the form

$$
v(H)=\min \left\{v_{0}(H), v_{1}(H)\right\} .
$$

Here $v_{0}$ comes from the standard connectivity range in the nonequivariant BlakersMassey theorem [9, Theorem 2.5] and it depends on the cocartesianity of the subcubes of $X$. The second term $v_{1}$ is purely equivariant and it depends on the connectivity of the maps of $X$. It is infinite if $J$ has the trivial $G$-action.

In particular, this Blakers-Massey theorem expresses the fact that the identity functor on pointed $G$-spaces is $G-1$-analytic in the sense of equivariant calculus of functors as defined in [6]; see Example 2.5. The Blakers-Massey theorem has a dual form, which we prove in Theorem 2.6. In the same way that the Freudenthal suspension theorem is an immediate consequence of the Blakers-Massey theorem for the square

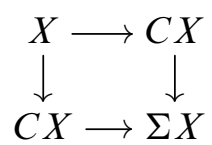

the equivariant Freudenthal suspension theorem follows from the equivariant BlakersMassey theorem applied to a certain equivariant cube, where all but the initial and the final vertices are contractible. Given a finite $G$-set $J$ and a pointed $G$-space $X$, let $\Sigma^{J} X$ and $\Omega^{J} X$ be the suspension and the loop space of $X$ by the permutation representation of $J$, respectively. We prove the following corollary in Section 2.2. 
Corollary (Equivariant suspension theorem [16]) Let $X$ be a well-pointed $G$-space. Then the unit of the $\left(\Sigma^{J}, \Omega^{J}\right)$-adjunction restricted to $G$-fixed points $\eta: X^{G} \rightarrow$ $\left(\Omega^{J} \Sigma^{J} X\right)^{G}$ is $m$-connected, where

$$
m=\min \left\{2 \operatorname{Conn} X^{G}+1, \min _{H<G, J / H \neq J / G} \operatorname{Conn} X^{H}\right\} .
$$

As a second application of the equivariant Blakers-Massey theorem, we prove an equivariant version of the multiple disjunction theorem of [10] for equivariant configuration spaces. Let $M$ be a manifold with a proper $G$-action. The space of configurations of $J$-points in $M$ is the space $\operatorname{Conf}(J, M)$ of injective maps $J \succ M$. This space inherits a $G$-action by conjugation, and it is functorial in the $J$-variable with respect to injective $G$-maps. The following is proved in Section 2.3.

Corollary Let $J$ be a finite $G$-set and let $J_{+}$be the $G$-set $J$ with an added fixed basepoint. The diagram $\operatorname{Conf}\left(J_{+} \backslash(-), M\right): \mathcal{P}\left(J_{+}\right) \rightarrow$ Top has a canonical $G$-structure, and it is $v$-cartesian for the function

$$
v(H)=\min \left\{|J| m_{H}-2|J / H|+1, \min _{L<H, J / L \neq J / H}\left(m_{L}-|J / L|\right)\right\},
$$

where $m_{H}$ is the dimension of the fixed-points manifold $M^{H}$.

In fact, we prove a stronger statement involving suitably transverse families of submanifolds of $M$ (Theorem 2.8), and we deduce this corollary from the case where all these submanifolds are points. In Example 2.10, we show that, for $G=J=\mathbb{Z} / 2$, this range is sharp, and it is determined by the second term of the minimum.

We turn to another classical result in homotopy theory, the celebrated Theorem B of Quillen, from [17]. This theorem shows that, under certain conditions, the homotopy fiber of the geometric realization of a functor is itself the geometric realization of a category. It is not immediately clear how to generalize this result equivariantly. The analogous statement for an equivariant functor between categories with $G$-actions can easily be reduced to Theorem B by taking fixed points. In order to achieve an interesting equivariant Theorem $\mathrm{B}$, we need to extend it first to higher-dimensional cubes. Let $J$ be a finite $G$-set and let $\mathcal{P}_{0}(-): \mathcal{P}_{0}(J) \rightarrow$ Cat be the diagram of categories that sends a subset $U \subset J$ to $\mathcal{P}_{0}(U)$. Given a cube of categories $X: \mathcal{P}(J) \rightarrow$ Cat the natural transformations of diagrams from $\mathcal{P}_{0}(-)$ to the restriction of $X$ to $\mathcal{P}_{0}(J)$ form a category $\operatorname{Hom}\left(\mathcal{P}_{0}(-), X\right)$. A $G$-structure on $X$ induces a $G$-action on the category $\operatorname{Hom}\left(\mathcal{P}_{0}(-), X\right)$ by conjugation. There is an inclusion

$$
m_{\varnothing}: X_{\varnothing} \longrightarrow \operatorname{Hom}\left(\mathcal{P}_{0}(-), X\right)
$$


that sends an object $x$ of $X_{\varnothing}$ to the natural transformation $\mathcal{P}_{0}(U) \rightarrow X_{U}$ that sends every object of $\mathcal{P}_{0}(U)$ to $\iota_{*} x$, where $\iota_{*}: X_{\varnothing} \rightarrow X_{U}$ is induced by the inclusion $\varnothing \subset U$. Given a natural transformation $\Phi \in \operatorname{Hom}\left(\mathcal{P}_{0}(-), X\right)$, the over category $m_{\varnothing} / \Phi$ has an action of the stabilizer group $G_{\Phi}$. We prove the following result in Corollary 3.10.

Theorem Let $X: \mathcal{P}(J) \rightarrow$ Cat be a $J$-cube of categories that satisfies a certain "weak Reedy fibrancy condition" as in Definition 3.9. For every natural transformation $\Phi$ the classifying space of the category $m_{\varnothing} / \Phi$ is $G_{\Phi}$-equivalent to the total homotopy fiber over $B \Phi$ of the $J$-cube of spaces $B X$. In particular, if all the categories $m_{\varnothing} / \Phi$ are $G_{\Phi}$-contractible, $B X: \mathcal{P}(J) \rightarrow$ Top is a homotopy cartesian $J$-cube of spaces.

When $G$ is the trivial group and $J=1$ is the set with one element, this is precisely Quillen's Theorem B. If $J=2$ is the set with two elements, this is essentially Barwick and Kan's Quillen Theorem $B_{2}$ for homotopy pullbacks from [2]. To the best of the author's knowledge, this is a new result for larger $J$, even for the trivial group. The most prominent application of Quillen's Theorem B is probably Waldhausen's additivity theorem (see [22, Theorem 1.4.2]), which leads to the deloopings of algebraic $K-$ theory by the $S_{\bullet}$-construction. We expect the equivariant Theorem B to have similar applications in the equivariant algebraic $K$-theory of $G$-Waldhausen categories (as defined in [15]) and to ultimately lead to an $S_{\bullet}$-description of the deloopings of equivariant algebraic $K$-theory by the permutation representations of $G$.

The key for proving the equivariant Theorem B is to define a good model for the homotopy limit of a diagram of categories. Let $I$ be a small category with finite-dimensional nerve. Taking the over categories of $I$ defines a diagram of categories $I /_{(-)}: I \rightarrow C a t$. Given a functor $X: I \rightarrow C a t$, the natural transformations $\operatorname{Hom}\left(I /{ }_{(-)}, X\right)$ form a category whose nerve is the Bousfield-Kan formula for the homotopy limit of the nerve of $X$. The following is proved in Section 3.2, and its equivariant version in Section 3.3.

Theorem $\mathbf{B}^{I}$ Let $I$ be a category with finite-dimensional nerve, and let $X: I \rightarrow$ Cat be a "Reedy quasifibrant diagram" as in Definition 3.3. The classifying space $B \operatorname{Hom}\left(I /{ }_{(-)}, X\right)$ is the homotopy limit of the diagram of spaces $B X: I \rightarrow$ Top.

Outline The first section of the paper contains some preliminaries on equivariant diagrams. In Section 1.1 we introduce the notion of fixed-points diagrams, and in Sections 1.2 and 1.3 we study their interaction with homotopy colimits and limits, respectively. In Section 2.1 we prove the equivariant Blakers-Massey theorem. We apply it to the equivariant suspension Theorem in Section 2.2 and to equivariant configuration spaces in Section 2.3. Section 3.1 introduces a quasi-fibrancy condition on diagrams of categories, which we use in Section 3.2 to prove a higher dimensional Quillen's Theorem B. Section 3.3 contains its equivariant analogue. 


\section{Preliminaries on equivariant diagrams}

\subsection{Equivariant diagrams of spaces and their fixed points}

Let $G$ be a discrete group. A category with $G$-action is a functor $a: G \rightarrow C a t$, where the group $G$ is seen as a category with a unique object $*$. By abuse of notation we will refer to the underlying category $I=a(*)$ as a category with $G$-action.

Definition 1.1 [21] Let $I$ be a small category with $G$-action, and let $C$ be a possibly large category. A $G$-structure on a diagram $X: I \rightarrow C$ is a collection of natural transformations $\phi_{g}: X \rightarrow X \circ g$ for every $g$ in $G$, subject to the following axioms.

i) Let 1 be the unit of $G$. Then $\phi_{1}$ is the identity natural transformation on $X$.

ii) For every $h$ and $g$ in $G$, the diagram

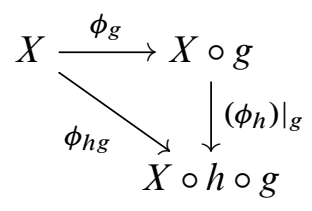

commutes. Here $\left.\left(\phi_{h}\right)\right|_{g}$ denotes the restriction of the natural transformation $\phi_{h}$ along the functor $g: I \rightarrow I$.

A diagram $X: I \rightarrow C$ equipped with a $G$-structure is called a $G$-diagram. A morphism of $G$-diagrams is a natural transformation of underlying diagrams $f: X \rightarrow Y$ such that the square

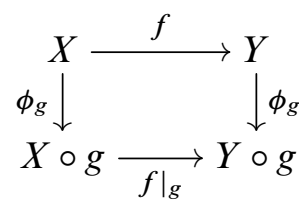

commutes for every $g$ in $G$. Here $\left.f\right|_{g}$ is the restriction of $f$ along the functor $g: I \rightarrow I$. The resulting category of $G$-diagrams is denoted $C_{a}^{I}$. We will often abuse the notation and write $g$ for the natural transformation $\phi_{g}$.

Example 1.2 Let $I$ and $J$ be two categories with $G$-action, and let $F: I \rightarrow J$ be an equivariant functor. The functor $F /(-): J \rightarrow$ Cat which sends an object $j$ to the over category $F / j$ has a natural $G$-structure. The natural transformation $\phi_{g}$ is defined by the functors $F / j \rightarrow F / g j$ that send an object $(i \in I, \alpha: F(i) \rightarrow j)$ to $(g i, g \alpha: F(g i)=g F(i) \rightarrow g j)$. By applying the classifying space functor, we obtain a $G$-diagram $B(F /-): J \rightarrow$ Top in the category of compactly generated Hausdorff spaces Top. 
The category of $G$-diagrams of spaces $\operatorname{Top}_{a}^{I}$ is enriched in the category of $G$-spaces Top $^{G}$. Given two $G$-diagrams $X$ and $Y$, the space of all natural transformations of underlying diagrams $\operatorname{Hom}_{I}(X, Y)$ inherits a $G$-action by conjugation:

$$
g \cdot f=\left(X \stackrel{\phi_{g-1}}{\longrightarrow} X \circ g^{-1} \stackrel{\left.f\right|_{g-1}}{\longrightarrow} Y \circ g^{-1} \stackrel{\phi_{g}}{\longrightarrow} Y\right) .
$$

The underlying set of the fixed-points space $\operatorname{Hom}_{I}(X, Y)^{G}$ is the set of morphisms of $G$-diagrams $\operatorname{Top}_{a}^{I}(X, Y)$.

The $G$-space $\operatorname{Hom}_{I}(X, Y)$ can be defined as a certain equalizer of $G$-spaces, and its construction can be dualized as follows. The $G$-action on $I$ induces a $G$-action on $I^{\mathrm{op}}$. Given a $G$-diagram $X: I \rightarrow$ Top and a $G$-diagram $Y: I^{\mathrm{op}} \rightarrow$ Top, we define the coequalizer of $G$-spaces

$$
X \otimes_{I} Y=\operatorname{colim}\left(\coprod_{\substack{\alpha: i \rightarrow j \\ \in \operatorname{hom} I}} Y_{i} \times X_{j} \rightrightarrows \coprod_{i \in \mathrm{Ob} I} Y_{i} \times X_{i}\right) .
$$

The maps are the standard maps of the Bousfield-Kan formula; see eg [11, Definition 18.3.2]. The $G$-action on the target of the maps sends $(y, x)$ in $Y_{i} \times X_{i}$ to $\left(\phi_{g}(y), \phi_{g}(x)\right)$ in $Y_{g i} \times X_{g i}$. The action on the source space is defined by a similar indexed coproduct.

Definition 1.3 Let $I$ be a category with $G$-action and $X \in \operatorname{Top}_{a}^{I}$ a $G$-diagram of spaces. The $G$-homotopy limit and the $G$-homotopy colimit of $X$ are the $G$-spaces respectively defined by

$$
\underset{I}{\operatorname{holim}} X=\operatorname{Hom}_{I}(B(I /-), X) \text { and } \underset{I}{\operatorname{hocolim}} X=X \otimes_{I}\left(B(-/ I)^{\mathrm{op}}\right) .
$$

The underlying space of the $G$-homotopy limit is the homotopy limit of the underlying diagram, defined via the Bousfield-Kan formula, and dually for the $G-$ homotopy colimit.

We recall that an equivariant map of $G$-spaces $f: X \rightarrow Y$ is an equivalence (in the fixed-points model structure) if its restriction to the $H$-fixed points $f: X^{H} \rightarrow Y^{H}$ is a weak homotopy equivalence of spaces for every subgroup $H$ of $G$. The vertex $X_{i}$ of a $G$-diagram $X$ inherits an action of the stabilizer group $G_{i}$ of the object $i \in I$, defined by the maps $\phi_{g}: X_{i} \rightarrow X_{g i}=X_{i}$.

Definition 1.4 [21] A morphism of $G$-diagrams $f: X \rightarrow Y$ is a weak equivalence if for every object $i$ of $I$ the map $f_{i}: X_{i} \rightarrow Y_{i}$ is an equivalence of $G_{i}$-spaces. 
The homotopical properties of the $G$-homotopy limit and of the $G$-homotopy colimit were studied extensively in [7]. In particular both constructions send equivalences of $G$-diagrams to equivalences of $G$-spaces.

Remark 1.5 Let $G \ltimes_{a} I$ be the Grothendieck construction of the functor $a$ : $G \rightarrow C a t$ which defines the $G$-action on $I$. A $G$-structure on a diagram $X: I \rightarrow$ Top is equivalent to an extension of $X$ to $G \ltimes_{a} I$ along the projection map $G \ltimes_{a} I \rightarrow I$. This results into an isomorphism of categories $\operatorname{Top}_{a}^{I} \cong \operatorname{Top}^{G \ltimes_{a} I}$; see [7, Lemma 1.9]. There is a relationship between the $G$-homotopy limit of a $G$-diagram $X \in \operatorname{Top}_{a}^{I}$, and the homotopy limit of the corresponding diagram $\bar{X}: G \ltimes_{a} I \rightarrow$ Top. The latter computes the homotopy fixed points of the former:

$$
\left.\operatorname{holim}_{G \ltimes_{a} I} \bar{X} \simeq \underset{I}{\operatorname{holim}} X\right)^{h G} .
$$

Dually, the homotopy colimit of $\bar{X}$ is equivalent to the homotopy orbits

$$
\left.\underset{G \ltimes_{a} I}{\operatorname{hocolim}} \bar{X} \simeq \underset{I}{(\operatorname{hocolim}} X\right)_{h G} .
$$

These equivalences are an immediate consequence of the Fubini Theorems [5, Proposition 26.5]. Homotopy orbits and homotopy fixed points are homotopy invariant with respect to naïve equivalences of $G$-spaces ( $G$-maps whose underlying map is an equivalence of spaces). We see from the formula above that $\operatorname{holim}_{G \ltimes_{a} I} \bar{X}$ and hocolim $_{G \ltimes_{a} I} \bar{X}$ are invariant for the pointwise naïve equivalences of $G$-diagrams. Thus the $G$-homotopy limit and the $G$-homotopy colimit retain more equivariant information than the homotopy limit and homotopy colimit of $\bar{X}$. The categorical (in opposition to homotopy) fixed points of the $G$-homotopy limits and colimits are the focus of the next two sections.

There is a notion of fixed points of a $G$-diagram. Let $H$ be a subgroup of $G$, and let $I^{H}$ be the subcategory of $I$ of objects and morphisms that are fixed (strictly) by the $H$-action. Equivalently, $I^{H}$ is the limit of the functor $H \rightarrow G \stackrel{a}{\rightarrow} C a t$. Since the $i$-vertex of a $G$-diagram $X \in \operatorname{Top}_{a}^{I}$ has an action of $G_{i}$, if $i$ belongs to $I^{H}$ the space $X_{i}$ has an $H$-action.

Definition 1.6 Let $H$ be a subgroup of $G$. The $H$-fixed-points diagram of a $G-$ diagram $X \in \operatorname{Top}_{a}^{I}$ is the diagram of spaces $X^{H}: I^{H} \rightarrow$ Top of pointwise fixed points $\left(X^{H}\right)_{i}=\left(X_{i}\right)^{H}$. Given a map $\alpha: i \rightarrow j$ in $I^{H}$, the corresponding map $X_{i}^{H} \rightarrow X_{j}^{H}$ is the restriction of $\alpha_{*}: X_{i} \rightarrow X_{j}$ on $H$-fixed points. 
The diagram $X^{H}: I^{H} \rightarrow$ Top is well defined on morphisms because for every $h$ in $H$ the diagram

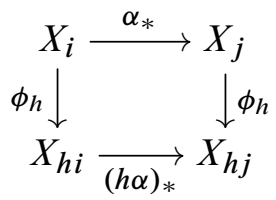

commutes by naturality of $\phi_{h}$. Hence if $\alpha$ is a morphism of $I^{H}$, the map $\alpha_{*}: X_{i} \rightarrow X_{j}$ is $H$-equivariant and it can be restricted on $H$-fixed points.

Remark 1.7 If $f: X \rightarrow Y$ is a morphism of $G$-diagrams, the map $f_{i}: X_{i} \rightarrow Y_{i}$ is $G_{i}$-equivariant for every object $i$ of $I$. Therefore $f$ restricts to a natural transformation $f^{H}: X^{H} \rightarrow Y^{H}$ for every subgroup $H$ of $G$. It is immediate from Definition 1.4 that $f$ is an equivalence of $G$-diagrams if and only if $f^{H}: X^{H} \rightarrow Y^{H}$ is an equivalence of $I^{H}$-shaped diagrams of spaces, for every subgroup $H$ of $G$. In this sense, the fixed-points diagrams of a $G$-diagram $X$ retain all the homotopical information of $X$.

\subsection{Equivariant homotopy colimits and fixed points}

Let $G$ be a discrete group, and let $a: G \rightarrow C a t$ be a $G$-action on a category $I=a(*)$. We study the interaction between the $G$-homotopy colimit of a $G$-diagram and its fixed-points diagrams, as in Definition 1.6.

Proposition 1.8 Suppose we have a $G$-diagram of spaces $X \in \operatorname{Top}_{a}^{I}$. Then there is a natural homeomorphism

$$
\underset{I}{(\operatorname{hocolim}} X)^{G} \cong \underset{I^{G}}{\operatorname{hocolim}} X^{G}
$$

Proof The coend hocolim $I X=X \otimes_{I}\left(B(-/ I)^{\mathrm{op}}\right)$ is homeomorphic to the geometric realization of the simplicial space with $n$-simplices

$$
\coprod_{i_{0} \leftarrow i_{1} \leftarrow \ldots \leftarrow i_{n}} X_{i_{n}}
$$

and the simplicial structure of [4, Chapter XII, $\S 5.1]$. The string of composable arrows under the coproduct is an element of $N_{n} I^{\mathrm{op}}$. The $G$-action on $X \otimes_{I}\left(B(-/ I)^{\mathrm{op}}\right)$ defined from the $G$-structure $\left\{\phi_{g}\right\}_{g \in G}$ of $X$ corresponds under this homeomorphism to the simplicial $G$-action

$$
g \cdot\left(i_{0} \leftarrow i_{1} \leftarrow \cdots \leftarrow i_{n}, x \in X_{i_{n}}\right)=\left(g i_{0} \leftarrow g i_{1} \leftarrow \cdots \leftarrow g i_{n}, \phi_{g}(x) \in X_{g i_{n}}\right) .
$$


Fixed points commute with geometric realizations; therefore, there exists a canonical homeomorphism

$$
(\underset{I}{\operatorname{hocolim} X} X)^{G} \cong\left|n \mapsto\left(\coprod_{\substack{i_{0} \leftarrow i_{1} \leftarrow \ldots \leftarrow i_{n} \\ \in N_{n} I^{\mathrm{op}}}} X_{i_{n}}\right)^{G}\right|,
$$

where the bars denote the geometric realization. Fixed points commute with $G-$ coproducts, in the sense that the above is homeomorphic to

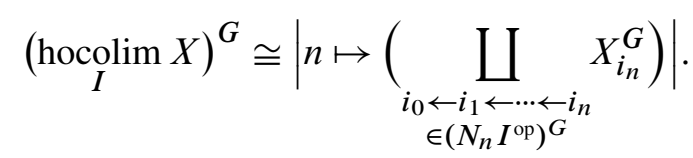

Observe that $\left(N_{n} I^{\mathrm{op}}\right)^{G}=N_{n}\left(I^{\mathrm{op}}\right)^{G}=N_{n}\left(I^{G}\right)^{\text {op }}$, and that for a $G$-fixed object $i_{n}$ the space $X_{i_{n}}$ has indeed a $G$-action. This geometric realization is homeomorphic to the homotopy colimit of the fixed-points diagram.

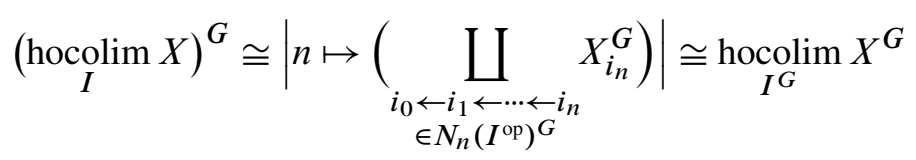

Remark 1.9 The description of the fixed points of the $G$-homotopy colimit in terms of fixed-points diagrams given in Proposition 1.8 makes it possible to deduce virtually all the equivariant homotopical properties of the $G$-homotopy colimit functor from the classical homotopical properties of the homotopy colimit. For example, it follows immediately from Proposition 1.8 that the $G$-homotopy colimits of equivalent $G-$ diagrams are equivalent as $G$-spaces, recovering [21, Theorem 6.1]. Another example is the equivariant Thomason's Theorem, proved as Corollary 1.10 below. We will see in Section 1.3 that the relationship between $G$-homotopy limits and fixed points is more involved.

An immediate application of Proposition 1.8 is the equivariant version of Thomason's Theorem. Let $X: I \rightarrow C a t$ be a $G$-diagram of categories. The Grothendieck construction $I$ \ $X$ has an induced $G$-action, defined on objects by

$$
g \cdot\left(i \in I, x \in \mathrm{Ob} X_{i}\right)=\left(g i, g x \in X_{g i}\right)
$$

and on morphisms by

$$
g \cdot\left(i \stackrel{\alpha}{\rightarrow} j, \alpha_{*} x \stackrel{\gamma}{\rightarrow} y\right)=\left(g i \stackrel{g \alpha}{\longrightarrow} g j,(g \alpha)_{*}(g x)=g\left(\alpha_{*} x\right) \stackrel{g \gamma}{\longrightarrow} g y\right) .
$$

The equality $(g \alpha)_{*}(g x)=g\left(\alpha_{*} x\right)$ expresses the naturality of $g: X \rightarrow X \circ g$. Thus the classifying space $B(I<X)$ inherits a $G$-action. The following result was proved in 
[7, Corollary 2.26] (see also [7, Remark 2.27]) as a special case of a general equivariant Fubini theorem. The proof presented here is more direct, reducing it to Thomason's Theorem [20] by a fixed-points argument.

Corollary 1.10 [7] Let $X: I \rightarrow C a t$ be a $G$-diagram of small categories, and let $B X$ be the $G$-diagram of spaces obtained by composing with the classifying space functor. There is a natural equivalence of $G$-spaces

$$
B(I<X) \stackrel{\simeq}{\longrightarrow} \underset{I}{\operatorname{hocolim}} B X .
$$

Proof The map $\eta_{X}: B(I<X) \rightarrow$ hocolim $_{I} B X$ defined in [20] is equivariant. For a subgroup $H$ of $G$, there is a natural isomorphism of categories $(I \succ X)^{H} \cong I^{H} \prec X^{H}$. Under this isomorphism and the homeomorphism of Proposition 1.8, the map $\eta_{X}^{H}$ corresponds to the map

$$
\eta_{X^{H}}: I^{H} 2 X^{H} \longrightarrow \underset{I^{H}}{\operatorname{hocolim}} B\left(X^{H}\right),
$$

which is an equivalence by Thomason's Theorem [20].

\subsection{Equivariant homotopy limits and fixed points}

The relationship between the fixed points of the $G$-homotopy limit and the homotopy limit of the fixed-points diagrams is more involved than it is for $G$-homotopy colimits. Given a pair of $G$-diagrams of spaces $K, X \in \operatorname{Top}_{a}^{I}$ there is a restriction map

$$
\operatorname{Hom}_{I}(K, X)^{G} \longrightarrow \operatorname{Hom}_{I^{G}}\left(K^{G}, X^{G}\right)
$$

that sends a morphism of $G$-diagrams to its restriction on the fixed-points diagrams. This is generally far from being an equivalence. When $K=B(I /-)$ and $I$ is of cubical shape we describe the homotopy fibers of the corresponding restriction map in Proposition 1.13 below. It turns out that in order to describe the whole fixed points space $\operatorname{Hom}_{I}(K, X)^{G}$ one needs to consider the natural transformations between the $H$-fixedpoints diagrams of $K$ and $X$, for every subgroup $H$ of $G$. These mapping spaces for the various subgroups of $G$ are related by means of the twisted arrow category.

Let us recall from [8] that the twisted arrow category $\operatorname{Tw}(C)$ of a category $C$ has as objects the morphisms $f: c \rightarrow d$ in $C$. A morphism $f \rightarrow f^{\prime}$ in $\operatorname{Tw}(C)$ is a commutative diagram

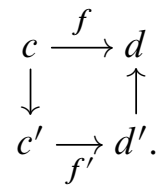


Let $\mathcal{O}_{G}$ be the orbit category of $G$. We use the twisted arrow category $\operatorname{Tw}\left(\mathcal{O}_{G}^{\text {op }}\right)$ to glue together the mapping spaces between the fixed-point diagrams of $K$ and $X$. An object of $\operatorname{Tw}\left(\mathcal{O}_{G}^{\text {op }}\right)$ is an equivariant map $G / H \stackrel{f}{\leftarrow} G / L$. This induces a functor $f^{*}: I^{H} \rightarrow I^{L}$, defined on objects by

$$
f^{*} i=f(L) \cdot i .
$$

A similar formula defines $f^{*}$ on morphisms. Precomposing a diagram with $f^{*}$ leads to a functor $f_{!}: \operatorname{Top}^{I^{L}} \rightarrow \operatorname{Top}^{I^{H}}$. Given two $G$-diagrams of spaces $K, X \in \operatorname{Top}_{a}^{I}$, we define a functor

$$
\operatorname{Tw}\left(\mathcal{O}_{G}^{\text {op }}\right)^{\text {op }} \longrightarrow \text { Top }
$$

by sending an object $G / H \stackrel{f}{\leftarrow} G / L$ of $\operatorname{Tw}\left(\mathcal{O}_{G}^{\text {op }}\right)$ to the space of natural transformations of $I^{H}$-diagrams $\operatorname{Hom}_{I^{H}}\left(K^{H}, f ! X^{L}\right)$. On morphisms this functor is defined as:

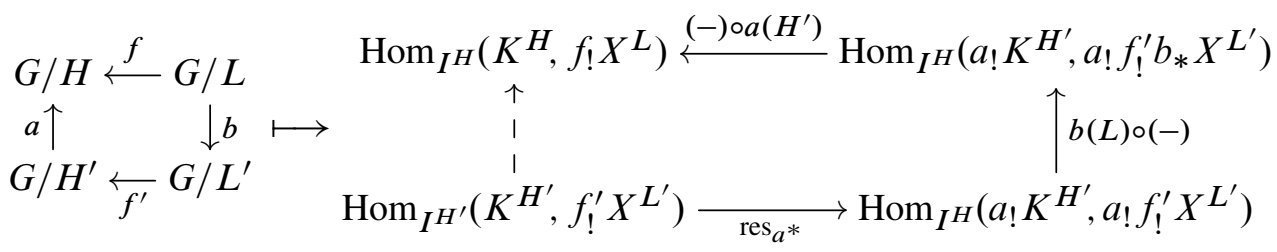

In the right-hand square, the lower horizontal map restricts a natural transformation along the functor $a^{*}: I^{H} \rightarrow I^{H^{\prime}}$. The top horizontal map is precomposition with pointwise multiplication by $a\left(H^{\prime}\right)$ in the $G$-structure on $K$, in symbols $a\left(H^{\prime}\right): K_{i}^{H} \rightarrow$ $K_{a(H) \cdot i}^{H}=\left(a_{*} K^{H}\right)_{i}$. Similarly, the right vertical map composes a natural transformation with the action of a representative of $b(L)$ for the $G$-structure of $X$. Explicitly, a natural transformation $\Phi: K^{H^{\prime}} \rightarrow f_{*}^{\prime} X^{L^{\prime}}$ is sent to

$$
K_{i}^{H} \stackrel{a\left(H^{\prime}\right)}{\longrightarrow} K_{a\left(H^{\prime}\right) \cdot i}^{H^{\prime}} \stackrel{\Phi_{a\left(H^{\prime}\right) i}}{\longrightarrow} X_{f^{\prime}\left(L^{\prime}\right) a\left(H^{\prime}\right) i}^{L^{\prime}} \stackrel{b(L)}{\longrightarrow} X_{b(L) f^{\prime}\left(L^{\prime}\right) a\left(H^{\prime}\right) i}^{L}=X_{f(L) i}^{L} .
$$

Proposition 1.11 Suppose we have a pair of $G$-diagrams $K, X$ in Top $I$. Then there is a natural homeomorphism

$$
\operatorname{Hom}_{I}(K, X)^{G} \cong \lim _{\substack{f: G / L \rightarrow G / H \\ \in \operatorname{Tw}\left(\mathcal{O}_{G}^{\mathrm{op}}\right)^{\text {op }}}} \operatorname{Hom}_{I^{H}}\left(K^{H}, f_{!} X^{L}\right) .
$$

In particular, when $K=B(I /-)$ this is a homeomorphism

$$
(\operatorname{holim} X)_{I}^{G} \cong \lim _{\substack{f: G / L \rightarrow G / H \\ \in \operatorname{Tw}\left(\mathcal{O}_{G}^{\mathrm{op}}\right)^{\text {op }}}} \operatorname{holim}_{I^{H}} f_{!} X^{L} .
$$

Proof In trying to dualize the argument of the proof of Proposition 1.8, one encounters the problem that $G$-indexed products do not commute with fixed points. Instead, 
we express the fixed points of the mapping space as a mapping space on a more complicated category. The functor $a: G \rightarrow C a t$ which defines the $G$-action on $I$ induces a functor $\bar{a}: \mathcal{O}_{G}^{\text {op }} \rightarrow$ Cat that sends $G / H$ to the fixed-points category $I^{H}$, and a $G$-map $G / H \stackrel{f}{\leftarrow} G / L$ to the functor $f^{*}: I^{H} \rightarrow I^{L}$ described above. Recall the isomorphism of categories $\operatorname{Top}_{a}^{I} \cong \operatorname{Top}^{G \ltimes_{a} I}$ of Remark 1.5 , where $G \ltimes_{a} I$ is the Grothendieck construction $G \prec a$. The canonical inclusion $G \rightarrow \mathcal{O}_{G}^{\text {op }}$ induces a functor $\operatorname{Top}_{G}^{\mathcal{O}_{G}^{\circ p}} \backslash \bar{a} \rightarrow \operatorname{Top}^{G}\left\{a \cong \operatorname{Top}_{a}^{I}\right.$. By [7, Theorem 2.28], this functor is the left adjoint of a Quillen equivalence

$$
L: \operatorname{Top}^{\mathcal{O}_{G}^{\mathrm{op}}}\left\langle\bar{a} \rightleftarrows \operatorname{Top}^{G \imath a} \cong \operatorname{Top}_{a}^{I}: R,\right.
$$

where the left-hand category has the projective model structure, and the right-hand category has a model structure where the equivalences are the equivalences of $G-$ diagrams of Definition 1.4. This is a sort of Elmendorf Theorem for the category of $G$-diagrams. The functor $R$ sends a $G$-diagram $X$ to the diagram with vertices

$$
R(X)_{\left(G / H, i \in I^{H}\right)}=X_{i}^{H} .
$$

The counit of this adjunction is an isomorphism; therefore, by [7, Theorem 2.28], we have a homeomorphism

$$
\operatorname{Hom}(K, X)^{G} \cong \operatorname{Hom}_{\mathcal{O}_{G}^{\mathrm{op}}}(\bar{a}(R(K), R(X)) .
$$

We finish the proof by defining a homeomorphism between the right-hand side and the limit of Proposition 1.11. An element of $\operatorname{Hom}_{\mathcal{O}_{G} \mathrm{op}} \mathrm{a}(R(K), R(X))$ is the data of a map $\Phi_{(H, i)}: K_{i}^{H} \rightarrow X_{i}^{H}$ for every subgroup $H$ of $G$ and fixed object $i \in I^{H}$, subject to compatibility conditions corresponding to the morphisms of $\mathcal{O}_{G}^{\mathrm{op}}>\bar{a}$. For every equivariant map $G / H \stackrel{f}{\leftarrow} G / L$ define a map

$$
\operatorname{Hom}_{\mathcal{O}_{G}^{\mathrm{op}}}\left\langle\bar{a}(R(K), R(X)) \longrightarrow \operatorname{Hom}_{I^{H}}\left(K^{H}, f_{!} X^{L}\right)\right.
$$

by sending a collection of maps $\Phi$ as above to

$$
K_{i}^{H} \stackrel{\Phi_{(H, i)}}{\longrightarrow} X_{i}^{H} \stackrel{f(L)}{\longrightarrow} X_{f(L) \cdot i}^{L}=\left(f_{!} X^{L}\right)_{i} .
$$

Naturality of $\Phi$ insures that these maps are compatible with the morphisms of the twisted arrow category, and this defines a map

$$
\operatorname{Hom}_{\mathcal{O}_{G}^{\mathrm{op}} \bar{a}}(R(K), R(X)) \longrightarrow \lim _{f \in \operatorname{Tw}\left(\mathcal{O}_{G}^{\mathrm{op}}\right)^{\mathrm{op}}} \operatorname{Hom}_{I^{H}}\left(K^{H}, f_{!} X^{L}\right) .
$$

The inverse of this map sends a collection of natural transformations $\Psi_{f}: K^{H} \rightarrow f_{!} X^{L}$ in the limit to the collection of maps

$$
\Psi_{\operatorname{id}_{G / H}}: K_{i}^{H} \longrightarrow X_{i}^{H} .
$$


Remark 1.12 The limit over the twisted arrow category of Proposition 1.11 is in general not a homotopy limit, and hence it is not homotopy invariant. This prevents us from easily deduce homotopical properties of the equivariant mapping space $\operatorname{Hom}_{I}(K, X)$ from the homotopical properties of the fixed-points diagrams. For example, proving that the $G$-homotopy limit functor preserves equivalences requires a considerable amount of work. The equivariant mapping space $\operatorname{Hom}_{I}(K, X)$ does however enjoy many equivariant homotopical properties, which are studied extensively in [7] using a model-categorical approach.

We turn our attention to the connectivity of the restriction map $\left(\operatorname{holim}_{I} X\right)^{G} \rightarrow$ $\operatorname{holim}_{I^{G}} X^{G}$ when $I$ has the shape of a punctured cube. This will play a key role in the proof of the equivariant Blakers-Massey Theorem 2.3. Let $J$ be a finite $G$-set, and let $\mathcal{P}_{0}(J)$ be the poset of nonempty subsets of $J$ ordered by inclusion. A group element $g$ acts on this poset by sending a subset $U$ to the image $g(U)$ by the map $g: J \rightarrow J$. A $G$-diagram $X \in \operatorname{Top}_{a}^{\mathcal{P}_{0}(J)}$ is called a punctured $J$-cube. Observe that there is an isomorphism of posets $\mathcal{P}_{0}(J)^{G} \cong \mathcal{P}_{0}(J / G)$, and that the inclusion $\mathcal{P}_{0}(J)^{G} \rightarrow \mathcal{P}_{0}(J)$ corresponds to the preimage functor $p^{-1}: \mathcal{P}_{0}(J / G) \rightarrow \mathcal{P}_{0}(J)$ associated to the quotient map $p: J \rightarrow J / G$. This induces a commutative square of spaces

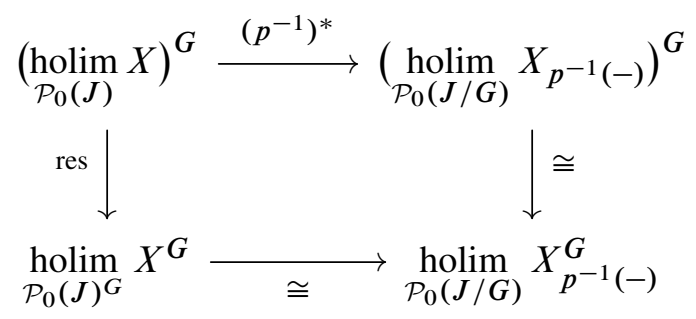

for every punctured $J$-cube $X \in \operatorname{Top}_{a}^{\mathcal{P}_{0}}{ }^{(J)}$. The right vertical map is an isomorphism because $G$ acts trivially on $\mathcal{P}_{0}(J / G)$ and because homotopy limits commute with fixed points. It is then sufficient to understand the restriction along the preimage functor.

Let $p: J \rightarrow S$ be a surjective equivariant map of finite $G$-sets with corresponding preimage functor $p^{-1}: \mathcal{P}_{0}(S) \rightarrow \mathcal{P}_{0}(J)$. We denote the fiber of an element $s \in S$ by $J_{s}=p^{-1}(s)$.

Proposition 1.13 Let $p: J \rightarrow S$ be a surjective equivariant map, and let $X \in \operatorname{Top}_{a}^{\mathcal{P}_{0}(J)}$ be a punctured $J$-cube of spaces. For every natural transformation $* \rightarrow X_{p^{-1}(-)}$ in $\operatorname{Top}_{a}^{\mathcal{P}_{0}(S)}$ the homotopy fiber of the restriction map

$$
\left(p^{-1}\right)^{*}: \operatorname{holim}_{\mathcal{P}_{0}(J)} X \longrightarrow \operatorname{Polim}_{\mathcal{P}_{0}(S)} X_{p^{-1}(-)}
$$


over the constant natural transformation $B\left(\mathcal{P}_{0}(S) /-\right) \rightarrow * \rightarrow X_{p^{-1}(-)}$ is $G$-equivalent to the $G$-space

$$
\operatorname{holim}_{W \in \mathcal{P}_{0}(S)} \operatorname{holim}_{\underline{U} \in \prod_{s \in W} \mathcal{P}_{0}\left(J_{s}\right)} \operatorname{hof}\left(X_{\amalg_{s \in W} U_{s}} \rightarrow X_{p^{-1}(W)}\right) .
$$

Proof The homotopy fiber $\operatorname{hof}\left(X_{\amalg_{s \in W} U_{s}} \rightarrow X_{p^{-1}(W)}\right)$ is taken over the basepoint of $X_{p^{-1}(W)}$ defined by natural transformation $* \rightarrow X_{p^{-1}(-)}$. Observe that since $* \rightarrow X_{p^{-1}(-)}$ is a map of $G$-diagrams the homotopy fibers form indeed a $G$-diagram. Let us define a $G$-equivariant functor

$$
\delta: \mathcal{P}_{0}(S) \times \prod_{s \in S} \mathcal{P}_{0}\left(J_{S}\right) \longrightarrow \mathcal{P}_{0}(J)
$$

by $\delta(W, \underline{U})=\amalg_{s \in W} U_{s}$. It is proved in [7, Proposition A.5] that this functor is equivariantly left-cofinal. Thus the horizontal map in the triangle

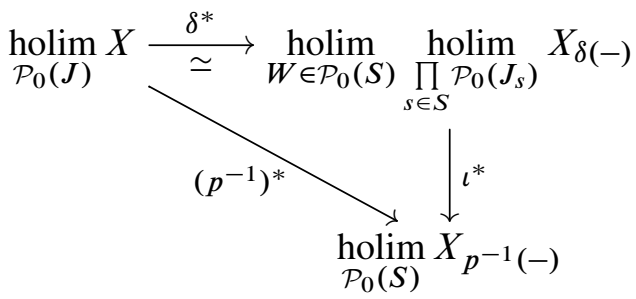

is a $G$-equivalence. Here $\iota: \mathcal{P}_{0}(S) \rightarrow \mathcal{P}_{0}(S) \times \prod_{s \in S} \mathcal{P}_{0}\left(J_{S}\right)$ is the inclusion that sends $W$ to the pair $\left(W,\left\{J_{s}\right\}_{s \in S}\right)$. The homotopy fibers of $\left(p^{-1}\right)^{*}$ and $\iota^{*}$ are therefore equivalent. Since homotopy limits and homotopy fibers commute, the homotopy fiber of $\iota^{*}$ is equivalent to

$$
\operatorname{holim}_{W \in \mathcal{P}_{0}(S)} \operatorname{holim}_{\underline{U} \in \prod_{s \in S} \mathcal{P}_{0}\left(J_{S}\right)} \operatorname{hof}\left(X_{\delta(W, \underline{U})} \rightarrow X_{p^{-1}(W)}\right) .
$$

Finally observe that for every fixed $W$ the functor $\delta(W,-)$ does not depend on the components of $\underline{U}$ indexed over the orbits in $J / G-W$.

Let us calculate the connectivity of this homotopy fiber for the quotient map $p: J \rightarrow$ $J / G$. For any subset $U$ of $J$ we let $\langle U\rangle_{G}=p^{-1} p(U)$ be the smallest $G$-invariant subset of $J$ which contains $U$.

Corollary 1.14 Let $J$ be a finite $G$-set and let $X: \mathcal{P}_{0}(J) \rightarrow$ Top be a $G$-diagram of spaces. Suppose that the composition of the canonical maps

$$
\left(\lim _{\mathcal{P}_{0}(J)} X\right)^{G} \rightarrow\left(\operatorname{holim}_{\mathcal{P}_{0}(J)} X\right)^{G} \rightarrow \operatorname{holim}_{\mathcal{P}_{0}(J)^{G}} X^{G}
$$


is surjective in $\pi_{0}$. Then the restriction map $\left(\operatorname{holim}_{\mathcal{P}_{0}(J)} X\right)^{G} \rightarrow \operatorname{holim}_{\mathcal{P}_{0}(J)^{G}} X^{G}$ is at least $m$-connected, where

$$
m=\min _{\substack{H<G \\ J / H \neq J / G}} \min _{U \in \mathcal{P}_{0}(J)^{H}}\left(\operatorname{Conn}\left(X_{U}^{H} \rightarrow X_{\langle U\rangle_{G}}^{H}\right)-|U / H|\right)+1 .
$$

Remark 1.15 The $\pi_{0}$ hypothesis of Corollary 1.14 is satisfied if for every fixed object $V$ in $\mathcal{P}_{0}(J)^{G}$ the connectivity of $X_{V}^{G}$ is greater or equal to $|V / G|-1$, since in this case holim $\operatorname{P}_{\mathcal{P}_{0}(J)^{G}} X^{G}$ is connected; see Proposition A.1.

Proof of Corollary 1.14 The $\pi_{0}$ assumption insures that all the homotopy fibers of the restriction are homotopy equivalent to the homotopy fiber over some point of $\operatorname{holim}_{\mathcal{P}_{0}(J)^{G}} X^{G}$ which is induced by a map of $G$-diagrams $* \rightarrow X_{p^{-1}(-)}$, as in Proposition 1.13. The formula for the connectivity of equivariant homotopy limits of Proposition A.1 shows that the homotopy fibers are at least

$$
\begin{aligned}
& \min _{(W, \underline{U})}\left(\operatorname{Conn} \operatorname{hof}\left(X_{\amalg_{o \in W} U_{o}}^{H} \rightarrow X_{p^{-1}(W)}^{H}\right)\right. \\
& H \leq G_{(W, \underline{U})} \\
& \left.-\operatorname{dim}\left(\mathcal{P}_{0}(J / G) / W\right)-\sum_{o \in W} \operatorname{dim}\left(\mathcal{P}_{0}\left(J_{o}\right)^{H} / U_{o}\right)\right)
\end{aligned}
$$

connected, where $J_{o}=p^{-1}(\{o\})$ is the orbit $o$ considered as a subset of $J$. The sum of the dimensions of the over categories is

$$
|W|-1+\sum_{o \in W}\left(\left|U_{o} / H\right|-1\right)=-1+\sum_{o \in W}\left|U_{o} / H\right| .
$$

Observe that the expression $\bigsqcup_{o \in W} U_{o}$ defines an equivariant bijection between the pairs of subsets $\left(W \in \mathcal{P}_{0}(J / G), \underline{U} \in \prod_{o \in W} \mathcal{P}_{0}\left(J_{o}\right)\right)$ and the nonempty subsets of $J$, with inverse sending $U$ to $\left(p(U),\left\{U \cap J_{o}\right\}_{o \in p(U)}\right)$. Thus the homotopy fiber of the restriction is as connected as

$$
\min _{U \in \mathcal{P}_{0}(J)} \min _{H \leq G_{U}}\left(\operatorname{Conn} \operatorname{hof}\left(X_{U}^{H} \rightarrow X_{p^{-1} p(U)}^{H}\right)+1-|U / H|\right) .
$$

By definition $p^{-1} p(U)=\langle U\rangle_{G}$, and the connectivity of a map is the connectivity of its homotopy fiber plus one. By switching the minimums in the formula above we see that the restriction map is

$$
\min _{H \leq G} \min _{U \in \mathcal{P}_{0}(J)^{H}}\left(\operatorname{Conn}\left(X_{U}^{H} \rightarrow X_{\langle U\rangle_{G}}^{H}\right)-|U / H|\right)+1
$$

connected. Finally observe that if the projection $J / H \rightarrow J / G$ is an isomorphism $U=\langle U\rangle_{G}$, and the homotopy fiber of

$$
X_{U}^{H} \rightarrow X_{\langle U\rangle_{G}}^{H}
$$


is contractible. Thus the groups $H$ with $J / H=J / G$ do not contribute to the minimum.

\section{The equivariant Blakers-Massey theorem and applications}

\subsection{The equivariant Blakers-Massey theorem}

Let $G$ be a discrete group and let $J$ be a finite $G$-set. We write $\mathcal{P}(J)$ for the poset category of subsets of $J$ ordered by inclusion. The $G$-action on $J$ induces a $G$-action on the category $\mathcal{P}(J)$, by sending a subset $U$ of $J$ to its image $g(U)$ under the map $g: J \rightarrow J$. This action restricts to the subposets

$$
\mathcal{P}_{0}(J)=\mathcal{P}(J) \backslash \varnothing, \quad \mathcal{P}_{1}(J)=\mathcal{P}(J) \backslash J .
$$

A $J$-cube of spaces is a $G$-diagram $X: \mathcal{P}(J) \rightarrow$ Top. The initial and final vertices $X_{\varnothing}$ and $X_{J}$ have $G$-actions induced by the $G$-structure of $X$, since $\varnothing$ and $J$ are fixed objects of $\mathcal{P}(J)$. Moreover the canonical maps

$$
X_{\varnothing} \longrightarrow \underset{\mathcal{P}_{0}(J)}{\operatorname{holim}} X, \quad \underset{\mathcal{P}_{1}(J)}{\operatorname{hocolim}} X \longrightarrow X_{J}
$$

are $G$-equivariant with respect to the $G$-actions on the homotopy limit and on the homotopy colimit of Section 1.1.

Definition 2.1 Let $J$ be a finite $G$-set and let $X \in \operatorname{Top}_{a}^{\mathcal{P}(J)}$ be a $J$-cube. Given a function $v:\{H \leq G\} \rightarrow \mathbb{N}$ which is invariant on conjugacy classes, we say that $X$ is $v$-cartesian if for every subgroup $H$ of $G$ the map of spaces

$$
X_{\varnothing}^{H} \longrightarrow\left(\underset{\mathcal{P}_{0}(J)}{\operatorname{holim}} X\right)^{H}
$$

is $v(H)$-connected. We say that $X$ is homotopy cartesian if it is $v$-cartesian for every function $v$. Dually, we say that $X$ is $v$-cocartesian if

$$
\left.\underset{\mathcal{P}_{1}(J)}{\operatorname{hocolim}} X\right)^{H} \longrightarrow X_{J}^{H}
$$

is $v(H)$-connected for every subgroup $H$ of $G$, and that $X$ is homotopy cocartesian if it is $v$-cocartesian for every $v$.

Remark 2.2 There is a natural isomorphism of categories $\mathcal{P}(J)^{H} \cong \mathcal{P}(J / H)$. Under this isomorphism the fixed-points diagram $X^{H}: \mathcal{P}(J)^{H} \rightarrow$ Top of a $J$-cube $X$ is a $J / H$-cube. By the fixed-points description of the $G$-homotopy colimit of Proposition 1.8, a $J$-cube $X$ is $v$-cocartesian precisely when the $J / H$-cube $X^{H}$ is 
$v(H)$-cocartesian for every subgroup $H$ of $G$. Because of the failure of $G$-homotopy limits to commute with fixed points, the analogous statement does not hold for cartesian $J$-cubes; compare Proposition 1.13.

Given a finite $G$-set $J$ and a subgroup $H$ of $G$ we denote by $\operatorname{Part}_{H}(J)$ the set of partitions of $J$ by $H$-subsets. This is the set of coverings $\left\{T_{\alpha} \subset J\right\}_{\alpha}$ of $J$ by nonempty $H$-invariant subsets such that $T_{\alpha}$ and $T_{\alpha^{\prime}}$ are disjoint when $\alpha \neq \alpha^{\prime}$. For every subset $U$ of $J$ we let $\langle U\rangle_{H}$ be the smallest $H$-invariant subset of $J$ that contains $U$.

Theorem 2.3 Let $X \in \operatorname{Top}_{a}^{\mathcal{P}(J)}$ be a $J$-cube, and suppose that for every subgroup $H$ of $G$ and every nonempty $H$-subset $U \subset J$ the restriction $\left.X\right|_{\mathcal{P}(U)}$ is $v^{U}$-cocartesian for some function $v^{U}:\{K \leq H\} \rightarrow \mathbb{N}$. Suppose moreover that these functions satisfy $v^{U} \leq v^{V}$ whenever $U \subset V$ are nonempty $H$-subsets of $J$. Then $X$ is $v$-cartesian, where $v$ is the minimum $v=\min \left\{v_{0}, v_{1}\right\}$ of the functions

and

$$
v_{0}(H)=\min _{\left\{T_{\alpha}\right\} \in \operatorname{Part}_{H}(J)}\left(\sum_{\alpha} v^{T_{\alpha}}(H)\right)-|J / H|+1
$$

$$
v_{1}(H)=\min _{\substack{L<H \\ J / L \neq J / H}} \min _{U \in \mathcal{P}_{0}(J)^{L}}\left(\operatorname{Conn}\left(X_{U}^{L} \rightarrow X_{\langle U\rangle_{H}}^{L}\right)-|U / L|\right) .
$$

Remark 2.4 The first term of the minimum is analogous to the formula of Goodwillie's Blakers-Massey theorem [9, Theorem 2.5] for $n$-cubes. The second term is purely equivariant: if the $G$-action on $J$ is trivial the set of subgroups $L<H$ such that $J / L \neq J / H$ is empty and $v_{1}$ is infinite. If $J$ is the set with $n$ elements and the trivial $G$-action, this is the standard Blakers-Massey theorem for $n$-cubes of $G$-spaces.

Example 2.5 Given a well-pointed $G$-space $X$ and a finite $G$-set $J$, consider the $J$-cube of spaces $w^{J}(X)$ with vertices

$$
w^{J}(X)_{U}= \begin{cases}\bigvee_{J} X & \text { if } U=\varnothing, \\ X & \text { if } U=\{j\}, \\ * & \text { otherwise. }\end{cases}
$$

The map $w^{J}(X)_{\varnothing} \rightarrow w^{J}(X)_{\{j\}}$ is the pinch map that collapses the wedge summands not indexed by $j$. The $G$-structure on $w^{J}(X)$ is defined by the $G$-action on the indexed wedge at the initial object, and by the $G$-action maps $g: X \rightarrow X$ between the $\{j\}$ and the $\{g j\}$-vertex. For every $H$-subset $U$ of $J$ there is a natural $H$-equivalence

$$
\underset{\mathcal{P}_{1}(U)}{\operatorname{hocolim}} w^{J}(X) \stackrel{\simeq}{\longrightarrow} \Sigma^{\overline{J \backslash U}} X,
$$


where the target is the suspension by the reduced permutation representation of the complement $J \backslash U$ (with the convention that this suspension is the point when $U=J$ ). Hence the restricted cube $\left.w^{J}(X)\right|_{\mathcal{P}(U)}$ is

$$
\nu^{U}(H)=\operatorname{Conn} X^{H}+|J / H|-|U / H|
$$

cocartesian if $U$ is a proper subset of $J$, and cocartesian for $U=J$. The first term $v_{0}$ of the minimum of Theorem 2.3 is realized when the partition of $J$ has two sets, with value

$v_{0}(H)=2\left(\operatorname{Conn} X^{H}+|J / H|\right)-\left(\left|T_{1} / H\right|+\left|T_{2} / H\right|\right)-|J / H|+1=2 \operatorname{Conn} X^{H}+1$.

Since all the vertices corresponding to subsets of cardinality greater than one are contractible, the second term of the minimum of Theorem 2.3 is

$$
v_{1}(H)=\min _{\substack{L<H \\ J / L \neq J / H}} \min _{j \in J^{L}} \operatorname{Conn}\left(X^{L} \rightarrow *\right)-1 \geq \min _{L<H} \operatorname{Conn} X^{L} .
$$

Thus the cube $w^{J}(X)$ is $v$-cartesian, where $v$ is the function

$$
v(H)=\min \left\{2 \operatorname{Conn} X^{H}+1, \min _{L<H} \operatorname{Conn} X^{L}\right\} .
$$

The limit of $w^{J}(X)$ over $\mathcal{P}_{0}(J)$ is the indexed product of $J$-copies of $X$, and the Blakers-Massey theorem for $w^{J}(X)$ tells us that the inclusion

$$
w^{J}(X)_{\varnothing}=\bigvee_{J} X \longrightarrow \prod_{J} X=\operatorname{holim}_{\mathcal{P}_{0}(J)} w^{J}(X)
$$

is $v$-connected. This range together with the Blakers-Massey Theorem for the trivial $G$-sets $J=n$ express the fact that the identity functor on pointed $G$-spaces is $G-1-$ analytic in the sense of equivariant calculus of functors; see [6, Definition 2.3.1].

The Blakers-Massey Theorem 2.3 has the following dual statement.

Theorem 2.6 Let $X \in \operatorname{Top}_{a}^{\mathcal{P}(J)}$ be a $J$-cube, and suppose that for every subgroup $H$ of $G$ and for every nonempty $H$-subset $U \subset J$, the cube $\left.X\right|_{\mathcal{P}(U)}$ is $v^{U}$-cartesian. Moreover, suppose that the functions $v^{U}:\{K \leq H\} \rightarrow \mathbb{N}$ satisfy $v^{U} \leq v^{V}$ whenever $U \subset V$ are nonempty $H$-subsets of $J$. Then $X$ is $v$-cocartesian, for

$$
v(H)=\min _{\left\{T_{\alpha}\right\} \in \operatorname{Part}_{H}(J)}\left(|J / H|-1+\sum_{\alpha} \min \left\{v^{T_{\alpha}}(H), v_{1}(H)\right\}\right),
$$

where $v_{1}$ is the function of Theorem 2.3. 
Proof of Theorem 2.3 For each subgroup $H$ of $G$, we need to calculate the connectivity of the canonical map $\phi: X_{\varnothing}^{H} \rightarrow\left(\operatorname{holim}_{\mathcal{P}_{0}(J)} X\right)^{H}$ at any basepoint in $X_{\varnothing}^{H}$. Since the empty set is initial in $\mathcal{P}(J)^{H}$, a basepoint of $X_{\varnothing}^{H}$ defines a basepoint $* \rightarrow X^{H}$ for the whole fixed-points diagram. There is a commutative diagram

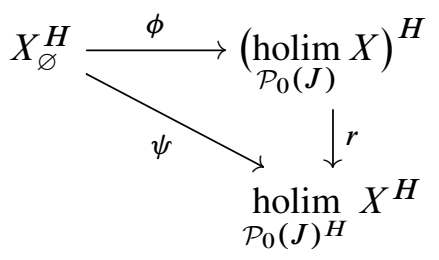

where $r$ is the restriction map of Proposition 1.13, and $\psi$ is the canonical map for the fixed-points diagram $X^{H}$. Our map is as connected as

$$
\text { Conn } \phi=\min \{\operatorname{Conn} \psi, \operatorname{Conn} r-1\} \text {. }
$$

The connectivity of $\psi$ expresses how cartesian the $J / H$-cube $X^{H}$ is, and it is determined by the standard Blakers-Massey theorem, [9, Theorem 2.5]. In order to apply this theorem we need to compare the cocartesianity of the subcubes of $X^{H}$. Under the canonical isomorphism $\mathcal{P}(J / H)=\mathcal{P}(J)^{H}$ a nonempty subset $U \subset J / H$ corresponds to a nonempty $H$-subsets of $J$. By assumption the cube $X^{H}$ is $v^{U}(H)-$ cocartesian, and for subsets $U \subset V \subset J / H$ the inequality $v^{U}(H) \leq v^{V}(H)$ holds. By [9, Theorem 2.5], the cube $X^{H}$ is

$$
\min _{\left\{T_{\alpha}\right\}}\left\{\sum_{\alpha} v^{T_{\alpha}}(H)\right\}-|J / H|+1
$$

cartesian, where $\left\{T_{\alpha}\right\}$ runs over the partitions of $J / H$, which correspond to the partitions of $J$ by $H$-subsets. This shows that $\psi$ is as connected as the function $v_{0}$ of the statement.

Now we calculate the connectivity of the restriction map $r$. We can assume that $\psi$ is at least 0 -connected, otherwise the connectivity range of Theorem 2.3 gives us no information about the cartesianity of $X$. The $\pi_{0}$-hypothesis of Corollary 1.14 is satisfied, since there is a commutative diagram

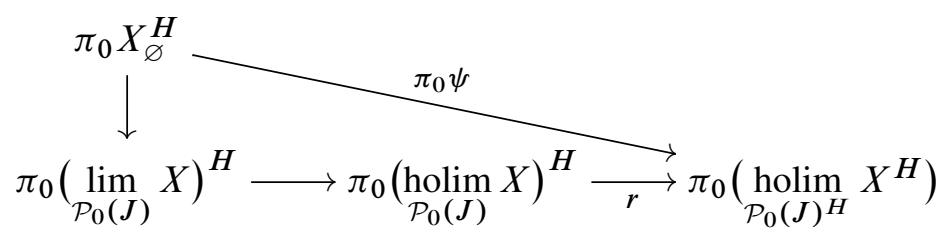


and the surjectivity of $\pi_{0} \psi$ implies the surjectivity of the lower composite. By Corollary 1.14 , the connectivity of $r$ is precisely $v_{1}$.

Proof of Theorem 2.6 For every subgroup $H$ of $G$ there is a commutative diagram

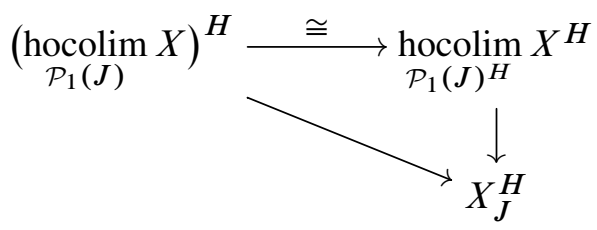

where the homeomorphism is from Proposition 1.8. Since $\mathcal{P}_{1}(J)^{H}$ is isomorphic to $\mathcal{P}_{1}(J / H)$, we need to determine how cocartesian the $J / H$-cube $X^{H}$ is. By the dual Blakers-Massey theorem [9, Theorem 2.6], it is

$$
\min _{\left\{T_{\alpha}\right\} \in \operatorname{Part}_{H}(J)}\left(|J / H|-1+\sum_{\alpha} \omega^{T_{\alpha}}(H)\right)
$$

cocartesian, if each restriction $\left.X^{H}\right|_{\mathcal{P}\left(T_{\alpha} / H\right)}$ is $\omega^{T_{\alpha}}(H)$-cartesian. To determine $\omega^{T_{\alpha}}(H)$, consider the commutative diagram

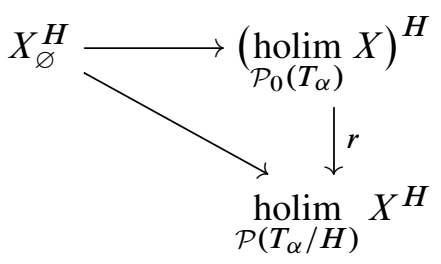

where the vertical map is the restriction. By hypothesis the horizontal map is $v^{T_{\alpha}}(H)-$ connected. The connectivity of $r$ is again given by Corollary 1.14.

\subsection{The equivariant Freudenthal suspension theorem}

Let $G$ be a discrete group and let $J$ be a finite $G$-set. We let $S^{J}$ be the permutation representation sphere of $J$, defined as the one-point compactification $\mathbb{R}[J]^{+}$of the $G$-vector space generated by $J$. Given a pointed $G$-space $X$, we define its $J$-loop and $J$-suspension respectively as the space of pointed maps and the smash product

$$
\Omega^{J} X=\operatorname{Map}_{*}\left(S^{J}, X\right), \quad \Sigma^{J} X=X \wedge S^{J}
$$

with $G$-action by conjugation on $\Omega^{J} X$ and diagonal on $\Sigma^{J} X$. The pair of functors $\Sigma^{J}: \operatorname{Top}_{*}^{G} \leftrightarrows \operatorname{Top}_{*}^{G}: \Omega^{J}$ is an adjunction. The equivariant Freudenthal suspension 
theorem of [16] (see also [13] and [1]) calculates the connectivity of the unit of this adjunction

$$
X^{G} \longrightarrow\left(\Omega^{J} \Sigma^{J} X\right)^{G}
$$

restricted to the $G$-fixed-points spaces. We show that this theorem is a direct consequence of the equivariant Blakers-Massey Theorem 2.3. The relationship between the Blakers-Massey theorem and the Freudenthal suspension theorem is well-established when $J$ has the trivial $G$-action.

Corollary 2.7 [16] Let $X$ be a well-pointed $G$-space. The unit of the $\left(\Sigma^{J}, \Omega^{J}\right)-$ adjunction $\eta: X^{G} \rightarrow\left(\Omega^{J} \Sigma^{J} X\right)^{G}$ is $m$-connected, where

$$
m=\min \left\{2 \operatorname{Conn} X^{G}+1, \min _{\substack{H<G \\ J / H \neq J / G}} \operatorname{Conn} X^{H}\right\} .
$$

Proof The idea of the proof is to construct the loop space $\Omega^{J} \Sigma^{J} X$ as the $G-$ homotopy limit of an equivariant cube. Let $J_{+}$be the $G$-set $J$ with an added fixed basepoint. Define a $J_{+}$-cube $\sigma^{J} X: \mathcal{P}\left(J_{+}\right) \rightarrow$ Top $_{*}$ with vertices

$$
\left(\sigma^{J} X\right)_{U}= \begin{cases}X & \text { if } U=\varnothing, \\ C^{U} X & \text { if } \varnothing \neq U \varsubsetneqq J_{+}, \\ \Sigma^{J} X & \text { if } U=J_{+},\end{cases}
$$

where $C^{U} X$ is the $U$-fold cone of $X$, defined as

$$
C^{U} X=\underset{\mathcal{P}(U)}{\operatorname{hocolim}}\left(V \longmapsto\left\{\begin{array}{ll}
X & \text { if } V=\varnothing, \\
* & \text { otherwise. }
\end{array}\right)\right.
$$

The functor $\sigma^{J} X$ has a natural $G$-structure. It is defined at the initial and final vertices by the $G$-actions on $X$ and $\Sigma^{J} X$ respectively, and at the other vertices by the canonical isomorphism

$$
C^{U} X \longrightarrow C^{g U} X
$$

induced by the functor $g: \mathcal{P}(U) \rightarrow \mathcal{P}(g U)$. For the group $G=\mathbb{Z} / 2$ and the $G$-set $J=\mathbb{Z} / 2$, this is the cube

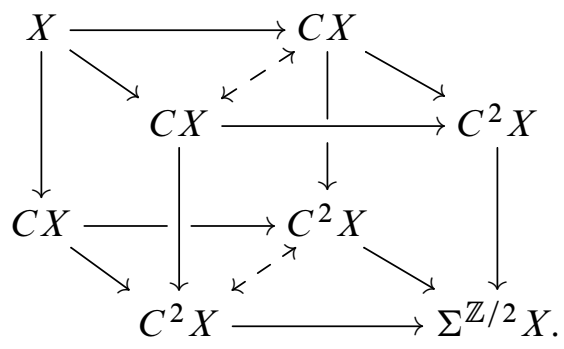


The dashed maps denote the $G$-structure between the nonfixed vertices. The space $C^{U} X$ is $G_{U}$-contractible, since for every subgroup $H$ of $G_{U}$, Proposition 1.8 provides a homeomorphism

$$
\left(C^{U} X\right)^{H} \cong C^{U / H}\left(X^{H}\right) \simeq * .
$$

This shows that the restriction of $\sigma^{J} X$ to $\mathcal{P}_{0}\left(J_{+}\right)$is equivalent to the $G$-diagram $\omega^{J} X: \mathcal{P}_{0}\left(J_{+}\right) \rightarrow$ Top defined by

$$
\left(\omega^{J} X\right)_{U}= \begin{cases}* & \text { if } U \neq J_{+} \\ \Sigma^{J} X & \text { if } U=J_{+}\end{cases}
$$

via the obvious pointed map $\left.\omega^{J} X \rightarrow \sigma^{J} X\right|_{\mathcal{P}_{0}\left(J_{+}\right)}$. The homotopy limit of $\omega^{J} X$ is $G$-homeomorphic to $\Omega^{J} \Sigma^{J} X$, by inspection on the definition of the Bousfield-Kan formula. This gives a commutative diagram of pointed $G$-spaces

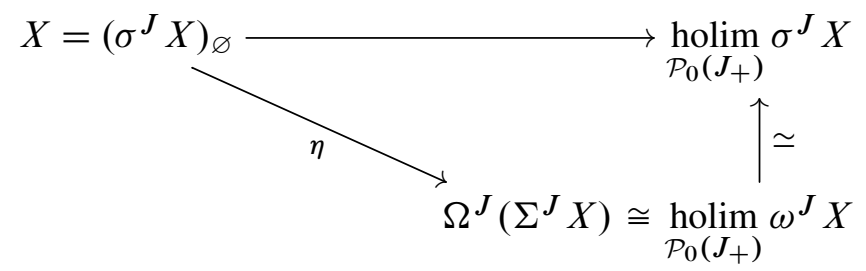

where the vertical map is a $G$-equivalence by the homotopy invariance of $G$-homotopy limits. The unit map $\eta$ is then as connected as $\sigma^{J} X$ is cartesian.

We show that $\sigma^{J} X$ satisfies the condition of the Blakers-Massey Theorem 2.3, and we show that its cartesianity estimate is the same as the range claimed in Corollary 2.7. For each subset $U$ of $J_{+}$, we need to find an estimate $v^{U}$ for the cocartesianity of the restriction $\left.\sigma^{J} X\right|_{\mathcal{P}(U)}$. For every subgroup $H$ of $G_{U}$, there are isomorphisms

$$
\left.\underset{\mathcal{P}_{1}(U)}{\operatorname{hocolim}} \sigma^{J} X\right)^{H} \cong \underset{\mathcal{P}_{1}(U / H)}{\operatorname{hocolim}}\left(\sigma^{J} X\right)^{H} \cong \underset{\mathcal{P}_{1}(U / H)}{\operatorname{hocolim}} \sigma^{J / H} X^{H}
$$

by the fixed-points description of Proposition 1.8. All the vertices of $\left.\sigma^{J / H} X^{H}\right|_{\mathcal{P}_{1}(U / H)}$ are contractible except for the initial one; thus there is a natural equivalence

$$
\left.\underset{\mathcal{P}_{1}(U)}{\operatorname{hocolim}} \sigma^{J} X\right)^{H} \cong \underset{\mathcal{P}_{1}(U / H)}{\operatorname{hocolim}} \sigma^{J / H} X^{H} \cong \Sigma^{|U / H|-1} X^{H} .
$$

Therefore, the canonical map $\left(\operatorname{hocolim}_{\mathcal{P}_{1}(U)} \sigma^{J} X\right)^{H} \rightarrow\left(\sigma^{J} X\right)_{U}^{H}$ factors through the equivalence

$$
\left.\underset{\mathcal{P}_{1}(U)}{\operatorname{hocolim}} \sigma^{J} X\right)^{H} \stackrel{\simeq}{\rightarrow} \Sigma^{|U / H|-1} X^{H} \rightarrow\left(\sigma^{J} X\right)_{U}^{H}= \begin{cases}C^{U / H} X^{H} & \text { if } U \neq J_{+} \\ \Sigma^{|J / H|} X^{H} & \text { if } U=J_{+}\end{cases}
$$


This map is (Conn $X^{H}+|U / H|$ )-connected for $U \neq J_{+}$and it is an equivalence for $U=J_{+}$. It follows that $\left.\sigma^{J} X\right|_{\mathcal{P}(U)}$ is $v^{U}$-cocartesian for the function

$$
v^{U}(H)= \begin{cases}\operatorname{Conn} X^{H}+|U / H| & \text { if } U \neq J_{+} \\ \infty & \text { if } U=J_{+}\end{cases}
$$

These functions satisfy $v^{U} \leq v^{V}$ for $H$-subsets $U \subset V$ of $J_{+}$, and the equivariant Blakers-Massey Theorem 2.3 applies. The term $v_{0}(G)$ in the range of Theorem 2.3 is

$$
\begin{aligned}
& \min _{\left\{T_{\alpha}\right\} \in \operatorname{Part}_{G}\left(J_{+}\right)}\left\{\sum_{\alpha} v^{T_{\alpha}}(G)\right\}-\left|J_{+} / G\right|+1 \\
& \quad=\min _{\left\{J_{+}\right\} \neq\left\{T_{\alpha}\right\}_{\alpha \in A}}\left\{\sum_{\alpha}\left(\left|T_{\alpha} / G\right|\right)+|A| \operatorname{Conn} X^{G}\right\}-\left|J_{+} / G\right|+1 .
\end{aligned}
$$

The trivial partition $\left\{J_{+}\right\}$is removed from the minimum because $v^{J_{+}}=\infty$. For any partition $\left\{T_{\alpha}\right\}$ of $J_{+}$by $G$-sets, the quotient $J_{+} / G$ decomposes as the disjoint union of the quotients $T_{\alpha} / G$. Therefore the sum in the formula above is equal to $\left|J_{+} / G\right|$. The minimum is thus realized when the size of the partition $|A|$ is minimal. This is the partition with two elements $\{J,+\}$, and the quantity above is

$$
\min _{\left\{J_{+}\right\} \neq\left\{T_{\alpha}\right\}_{\alpha \in A}}\left\{\left|J_{+} / G\right|+|A| \operatorname{Conn} X^{G}\right\}-\left|J_{+} / G\right|+1=2 \operatorname{Conn} X^{G}+1 .
$$

Since all but the initial and final vertices of $\sigma^{J} X$ are contractible the second term $v_{1}(G)$ of the minimum of Theorem 2.3 is

$$
\begin{aligned}
& \min _{\substack{H<G \\
J / H \neq J / G}} \min _{\substack{U \in \mathcal{P}_{0}\left(J_{+}\right)^{H} \\
U \neq J_{+}}}\left(\operatorname{Conn}\left(* \rightarrow \Sigma^{J / H} X^{H}\right)-|U / H|\right) \\
& \geq \min _{\substack{H<G \\
J / H \neq J / G}}\left(\operatorname{Conn} X^{H}+|J / H|-\left|J_{+} / H\right|+1\right) .
\end{aligned}
$$

\subsection{Equivariant intersections of submanifolds and configuration spaces}

Let $M$ be a manifold and let $P_{1}, \ldots, P_{n}$ be a collection of submanifolds of $M$. For every integer $2 \leq k \leq n$, we define inductively what it means for the collection $P_{1}, \ldots, P_{n}$ to be $k$-transverse in $M$.

(i) $P_{1}, \ldots, P_{n}$ is 2 -transverse if $P_{i}$ intersects $P_{j}$ transversely for every $i \neq j$.

(ii) $P_{1}, \ldots, P_{n}$ is $k$-transverse if it is $(k-1)$-transverse and if for every set $S \subset\{1, \ldots, n\}$ with $k$ elements and every $s \in S$ the submanifold $\bigcap_{t \in S \backslash s} P_{t}$ intersects $P_{S}$ transversely. 
Let $m$ be the dimension of $M$, and let $d_{i}$ be the dimension of $P_{i}$. If the collection of submanifolds $P_{1}, \ldots, P_{n}$ is $n$-transverse, the intersection $\bigcap_{s \in S} P_{s}$ is either a submanifold of $M$ of codimension $\sum_{s \in S}\left(m-d_{s}\right)$, or it is the empty submanifold.

Now suppose that $G$ is a discrete group, let $J$ be a finite $G$-set, and suppose that $G$ acts properly on a manifold $M$. Let $\left\{P_{j}\right\}_{j \in J}$ be a set of submanifolds indexed on $J$, and suppose that for every group element $g$ the corresponding automorphism of $M$ restricts to a map $g: P_{j} \rightarrow P_{g j}$. The $G$-action on $M$ defines a $G$-structure on the cube $M \backslash P_{\bullet}: \mathcal{P}(J) \rightarrow$ Top which sends a subset $U \subset J$ to the complement $M \backslash \bigcup_{j \notin U} P_{j}$. The $G$-structure is defined by the maps

$$
g: M \backslash \bigcup_{j \notin U} P_{j} \rightarrow M \backslash \bigcup_{j \notin U} g\left(P_{j}\right)=M \backslash \bigcup_{j \notin U} P_{g j}=M \backslash \bigcup_{j \notin g U} P_{j} .
$$

Theorem 2.8 Let $M$ be a manifold with a proper $G$-action, and let $\left\{P_{j}\right\}_{j \in J}$ be a set of closed submanifolds as above. Suppose moreover that for every $j \in J$ and every subgroup $H$ of $G$ the intersection $P_{j} \cap M^{H}$ is a submanifold of $M$, and that the collection of submanifolds $\left\{P_{j} \cap M^{H}\right\}_{j \in J}$ is $|J|$-transverse in $M^{H}$. Then the $J$-cube $M \backslash P_{\bullet}: \mathcal{P}(J) \rightarrow$ Top is $v$-cartesian, where $v$ is the function

$$
\min \left\{|J| m_{H}-2|J / H|+1-\sum_{j \in J} d_{j}(H), \min _{\substack{L<H \\ J / L \neq J / H}}\left(m_{L}-\max _{j \in J} d_{j}(L)-|J / L|\right)\right\} .
$$

Here $d_{j}(H)$ is the dimension of $P_{j} \cap M^{H}$, and $m_{H}$ is the dimension of $M^{H}$.

Proof In order to understand how cartesian $M \backslash P \bullet$ is it is sufficient, by the BlakersMassey theorem, to understand how cocartesian the subcubes of $M \backslash P_{\bullet}$ are. Let $H$ be a subgroup of $G$ and let $U$ be an $H$-invariant subset of $J$. The homotopy colimit of the restriction of $M \backslash P_{\bullet}$ to $\mathcal{P}_{1}(U)$ is $H$-equivalent to

$$
\underset{\mathcal{P}_{1}(U)}{\operatorname{hocolim}} M \backslash P_{\bullet} \stackrel{\simeq}{\rightarrow} \operatorname{colim}_{\mathcal{P}_{1}(U)} M \backslash P_{\bullet}=\left(M \backslash \bigcup_{j \notin U} P_{j}\right) \backslash \bigcap_{u \in U} P_{u} .
$$

Hence the cube $\left.\left(M \backslash P_{\bullet}\right)\right|_{\mathcal{P}(U)} ^{H}$ is $v^{U}(H)$-cocartesian, where $v^{U}(H)$ is the connectivity of the inclusion

$$
\nu^{U}(H)=\operatorname{Conn}\left(\left(M \backslash \bigcup_{j \notin U} P_{j}\right) \backslash \bigcap_{u \in U} P_{u} \longrightarrow M \backslash \bigcup_{j \notin U} P_{j}\right)^{H}
$$

of $H$-fixed points. This map is the inclusion of submanifolds

$$
\left(M^{H} \backslash\left(\bigcup_{j \notin U} P_{j}\right)^{H}\right) \backslash\left(\bigcap_{u \in U} P_{u}\right)^{H} \longrightarrow M^{H} \backslash\left(\bigcup_{j \notin U} P_{j}\right)^{H},
$$


which is as connected as the codimension of $\left(\bigcap_{u \in U} P_{u}\right)^{H}$ in $M^{H}$ minus one. By our transversality assumption $\left(\bigcap_{u \in U} P_{u}\right)^{H}=\bigcap_{u \in U} P_{u} \cap M^{H}$ is a submanifold of $M^{H}$ of codimension $\sum_{u \in U}\left(m_{H}-d_{u}(H)\right)$. The functions

$$
\nu^{U}(H)=\sum_{u \in U}\left(m_{H}-d_{u}(H)\right)-1
$$

satisfy $v^{U} \leq v^{V}$ when $U \subset V$ are both $H$-invariant, and Theorem 2.3 applies. The first term of the minimum of Theorem 2.3 is

$$
v_{0}(H)=\min _{\left\{T_{\alpha}\right\}_{\alpha \in A} \in \operatorname{Part}_{H}(J)}\left\{\sum_{\alpha \in A} \sum_{u \in T_{\alpha}}\left(m_{H}-d_{u}(H)\right)-|A|\right\}-|J / H|+1 .
$$

The double sum is independent of the partition, and this minimum is realized for the finest partition of $J$ by $H$-invariant sets. This is the partition of $J$ in $H$-orbits, and the term above is

$$
\sum_{j \in J}\left(m_{H}-d_{j}(H)\right)-2|J / H|+1 .
$$

The second term of the minimum of the Blakers-Massey formula is

$$
v_{1}(H)=\min _{\substack{L<H \\ J / L \neq J / H}} \min _{\substack{U \in \mathcal{P}_{0}(J)^{L} \\ U \neq J}}\left(\operatorname{Conn}\left(M \backslash \bigcup_{j \notin U} P_{j}\right)^{L} \rightarrow\left(M \backslash \bigcup_{j \notin\langle U\rangle_{H}} P_{j}\right)^{L}-|U / L|\right) .
$$

The connectivity of the inclusion above is $m_{L}-\max _{j \in\langle U\rangle_{H} \backslash U} d_{j}(L)-1$, and $v_{1}(H)$ is bounded by

$$
v_{1}(H) \geq \min _{\substack{L<H \\ J / L \neq J / H}}\left(m_{L}-\max _{j \in J} d_{j}(L)-1-|J / L|+1\right) .
$$

This theorem has interesting consequences for equivariant cubes of configuration spaces. What follows is an equivariant version of the multiple disjunction theorem [10] in the easy situation where the submanifolds $P_{j}$ are points. The techniques of [10] can supposedly be extended to equivariant collections of higher-dimensional submanifolds. Given a finite $G$-set $J$ and a proper $G$-manifold $M$, we let $\operatorname{Conf}(J, M)$ be the space of ordered configurations of $|J|$-points in $M$, with $G$ acting by conjugation. Explicitly, a configuration is an injective map $\underline{x}: J \succ M$, which is sent by the homeomorphism associated to $g \in G$ to the composite $g \underline{x} g^{-1}$. The cube $\operatorname{Conf}(J \backslash(-), M): \mathcal{P}(J) \rightarrow$ Top that sends a subset $U$ of $J$ to the configurations of $J \backslash U$-points in $M$ has a $G-$ structure defined by the maps $g: \operatorname{Conf}(J \backslash U, M) \rightarrow \operatorname{Conf}(J \backslash g U, M)$ which send $\underline{x}: J \backslash U \succ M$ to

$$
g \underline{x}: J \backslash g(U) \stackrel{g^{-1}}{\longrightarrow} J \backslash U \stackrel{\underline{x}}{\longrightarrow} M \stackrel{g}{\longrightarrow} M .
$$


Corollary 2.9 Let $J$ be a finite $G$-set and let $J_{+}$be the $G$-set $J$ with an added fixed basepoint. The $J_{+}-$cube $\operatorname{Conf}\left(J_{+} \backslash(-), M\right): \mathcal{P}\left(J_{+}\right) \rightarrow$ Top is $v$-cartesian, where $v$ is the function

$$
v(H)=\min \left\{|J| m_{H}-2|J / H|+1, \min _{\substack{L<H \\ J / L \neq J / H}}\left(m_{L}-|J / L|\right)\right\},
$$

and $m_{H}$ is the dimension of the fixed-points manifold $M^{H}$.

Because of the second term of the minimum, one cannot expect to make $v$ diverge by increasing the number of orbits of $J$, which is possible in the nonequivariant situation. If the action on $J$ is trivial, the second term is infinite and the range of Corollary 2.9 is the classical cartesianity range from embedding calculus; see [10]. Before proving Corollary 2.9, we see an example where the range is sharp and it is determined by the second term of the minimum.

Example 2.10 Let us consider the group $G=\mathbb{Z} / 2$ and the finite $G$-set $J=\mathbb{Z} / 2$ with action by left multiplication. The space $\operatorname{Conf}\left(\mathbb{Z} / 2_{+}, M\right)$ of configurations in a manifold with involution $M$ is the space of triples of pairwise distinct elements $\left(x_{+}, x_{0}, x_{1}\right)$ in $M$. Such a triple is sent by the nontrivial element of $\mathbb{Z} / 2$ to $\left(\tau x_{+}, \tau x_{1}, \tau x_{0}\right)$, where $\tau$ denotes the involution of $M$. The $\mathbb{Z} / 2$-fixed points of this space is described by a natural homeomorphism

$$
\operatorname{Conf}\left(\mathbb{Z} / 2_{+}, M\right)^{\mathbb{Z} / 2} \cong M^{\mathbb{Z} / 2} \times\left(M \backslash M^{\mathbb{Z} / 2}\right) .
$$

The equivariant cube $\operatorname{Conf}\left(\mathbb{Z} / 2_{+} \backslash(-), M\right)$ is the $\mathbb{Z} / 2_{+}$-cube

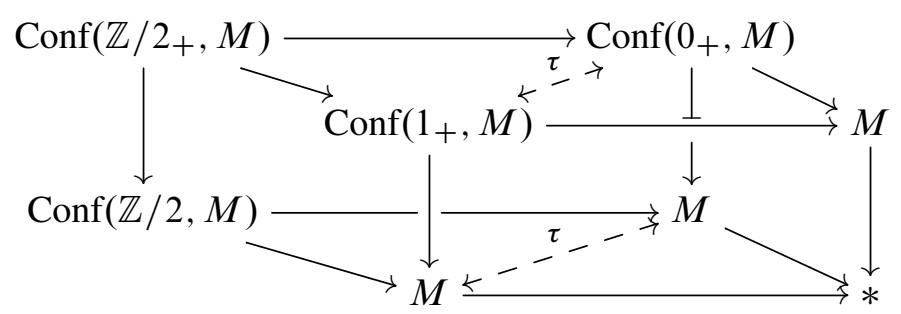

where 0 and 1 are the elements of $J=\mathbb{Z} / 2$. The dashed maps denote the $G$-structure on the nonfixed objects of $\mathcal{P}\left(\mathbb{Z} / 2_{+}\right)$. By Corollary 2.9 , the cartesianity of this cube on $\mathbb{Z} / 2$-fixed points is

$$
v(\mathbb{Z} / 2)=\min \left\{2 m_{\mathbb{Z} / 2}-1, m_{1}-2\right\} .
$$

Observe that since $m_{\mathbb{Z} / 2} \geq m_{1} / 2$ this quantity is always determined by the second term of the minimum $v(\mathbb{Z} / 2)=m_{1}-2$. 
Let us show that the estimate $v(\mathbb{Z} / 2)$ is in this case sharp. Let $\underline{x}=\left\{x_{0}, x_{1}, x_{+}\right\}$be a configuration which is fixed by the action, that is $x_{+}$belongs to $M^{\mathbb{Z} / 2}$ and $x_{1}=\tau\left(x_{0}\right)$. By taking homotopy fibers over $\underline{x}$ in the +- direction, we see that the cube above is as cartesian as the $\mathbb{Z} / 2-$ cube

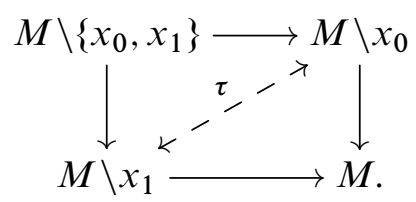

By inspection, a fixed point of $\operatorname{holim}\left(M \backslash x_{0} \rightarrow M \leftarrow M \backslash x_{1}\right)$ is the data of a path $\gamma: I \rightarrow M$ such that $\gamma(0) \neq x_{0}, \gamma(1) \neq x_{1}$ and $\tau \gamma(t)=\gamma(1-t)$. Such a path is determined by its restriction to the interval $[0,1 / 2]$, with the condition that the value $\gamma(1 / 2)$ belongs to $M^{\mathbb{Z} / 2}$. Thus the fixed points of this homotopy limit are homeomorphic to $\operatorname{holim}\left(M^{\mathbb{Z} / 2} \rightarrow M \leftarrow M \backslash x_{0}\right)$, and on $\mathbb{Z} / 2$-fixed points the square above is as cartesian as

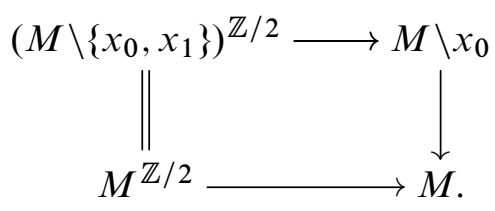

The vertical map is the identity because $x_{0} \neq x_{1}=\tau\left(x_{0}\right)$ is not fixed. This square is as cartesian as the connectivity of $M \backslash x_{0} \rightarrow M$ minus one, which is precisely

$$
\left(\operatorname{codim}\left(x_{0}\right)-1\right)-1=m_{1}-2=v_{1}(\mathbb{Z} / 2) .
$$

Proof of Corollary 2.9 Let $\underline{x}: J_{+} \succ M$ be a configuration. Then the collection of its restrictions $\underline{x}_{J_{+} \backslash U} \in \operatorname{Conf}\left(J_{+} \backslash U, M\right)$ defines a basepoint of the diagram $\operatorname{Conf}\left(J_{+} \backslash(-), M\right)$. Let $\mathcal{F}_{\underline{x}}(M): \mathcal{P}(J) \rightarrow$ Top be the $J$-cube defined by the homotopy fibers of the maps that forget the basepoint,

$$
\mathcal{F}_{\underline{x}}(M)_{U}=\operatorname{hof}\left(\operatorname{Conf}\left(J_{+} \backslash U, M\right) \longrightarrow \operatorname{Conf}(J \backslash U, M)\right),
$$

over the points $\underline{x}_{J \backslash U}$, with the induced canonical maps. The cube $\mathcal{F}_{x}(M)$ has a $G_{\underline{x}}$-structure, where $G_{\underline{x}}$ is the stabilizer group of the configuration $\underline{x}$, defined by the canonical maps

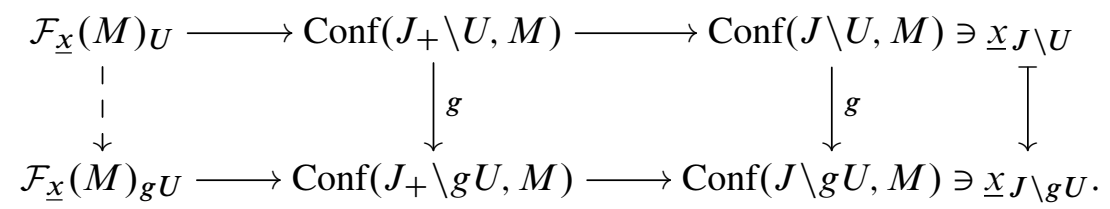


The homotopy fiber of the map $\operatorname{Conf}\left(J_{+}, M\right) \rightarrow \operatorname{holim}_{\mathcal{P}_{0}\left(J_{+}\right)} \operatorname{Conf}\left(J_{+} \backslash(-), M\right)$ over a configuration $\underline{x}$ is $G_{\underline{x}}$-equivalent to the homotopy fiber of the map $\mathcal{F}_{\underline{x}}(M)_{\varnothing} \rightarrow$ $\operatorname{holim}_{\mathcal{P}_{0}(J)} \mathcal{F}_{\underline{x}}(M)$ over the canonical basepoint defined by $\underline{x}$. Hence $v(H)$ is the minimum of the cartesianities of $\mathcal{F}_{\underline{x}}(M)$, for $\underline{x}$ running through the configurations in $\operatorname{Conf}\left(J_{+} \backslash U, M\right)$ which are fixed by $H$. The restriction map $\operatorname{Conf}\left(J_{+} \backslash U, M\right) \rightarrow$ $\operatorname{Conf}(J \backslash U, M)$ is a fibration of $G_{U}$-spaces, whose fiber over $\underline{x}_{J \backslash U}: J \backslash U \succ M$ is the manifold $M$ with the image of $\underline{x}_{J \backslash U}$ removed. Hence the cube $\mathcal{F}_{\underline{x}}(M)$ is equivalent as a $G_{\underline{x}}$-diagram to the $J$-cube

$$
F_{\underline{x}}(M)_{U}=M \backslash \bigcup_{j \in J \backslash U} x_{j} .
$$

The collection of 0 -dimensional submanifolds $\left\{x_{j}\right\}_{j \in J}$ satisfies the transversality conditions of Theorem 2.8, and the cube $F_{\underline{x}}(M)=M \backslash \underline{x}_{\bullet}$ is then $v(H)$-cartesian.

\section{The equivariant Quillen Theorem B}

Quillen's Theorem B of [17] shows that under certain conditions the homotopy fiber of the geometric realization of a functor $F: C \rightarrow D$ over an object $d$ in $D$ is weakly equivalent to the geometric realization of the over category $F / d$. It is not immediately clear how this result can be generalized to an equivariant context. The analogous statement for an equivariant functor between categories with $G$-actions can easily be reduced to Theorem B by taking fixed points. The generalization presented in this paper gives a categorical model for the total homotopy fiber of the geometric realization of a $J$-cube of categories. When $J$ is the set with one element, one recovers Quillen's Theorem B.

The key to our equivariant Theorem B is a categorical model for the $G$-homotopy limit of the nerve of a suitable $G$-diagram of categories. This result is not limited to diagrams of cubical shape, but it applies more generally to categories which satisfy a suitable finiteness condition; see Section 3.2. Even when $G$ is the trivial group our categorical model for the homotopy limit generalizes Theorem B considerably, from homotopy fibers to all finite homotopy limits. Since this result might be of interest to nonequivariant homotopy theorists we prove it as a separate statement.

The section is organized as follows. In Section 3.1, we define a categorical model for the homotopy limit of the nerve of a diagram $X: I \rightarrow C a t$, as well as a quasifibrancy condition analogous to the hypothesis of Quillen's Theorem B. In Section 3.2, we prove that the classifying space of the category constructed in Section 3.1 indeed models the homotopy limit of $B X$ if $X$ is quasifibrant. We call this result "Theorem $\mathrm{B}^{I}$ ". In 
Corollary 3.7, we use this result to give a categorical model for the total homotopy fiber of a cube of categories. We think of this corollary as a Theorem B for cubes. Section 3.3 contains the generalization of Theorem $\mathrm{B}^{I}$ to $G$-diagrams, and the equivariant Quillen Theorem B.

\subsection{Reedy quasifibrant diagrams of categories}

Let $I$ be a small category and let $K, X: I \rightarrow$ Cat be diagrams of small categories. The natural transformations from $K$ to $X$ form a category $\operatorname{Hom}(K, X)$. An object of this category is a natural transformation $\Phi: K \rightarrow X$, and a morphism $\Lambda: \Phi \rightarrow \Phi^{\prime}$ is a pair of natural transformations $\Lambda_{1}: K \rightarrow K$ and $\Lambda_{2}: X \rightarrow X$ such that the following square commutes:

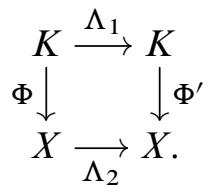

Remark 3.1 The category $\operatorname{Hom}(K, X)$ was introduced in [14] where the author shows, among other homotopical properties of this construction, that its nerve is isomorphic to the simplicial mapping space of natural transformations $\operatorname{Hom}(N K, N X)$. In particular, when $K$ is the functor $K=I /\left(_{-}\right): I \rightarrow C a t$, the nerve of $\operatorname{Hom}\left(I /{ }_{(-)}, X\right)$ is isomorphic to the Bousfield-Kan formula [4] for the homotopy limit of $N X$. This construction computes the homotopy limit of $N X$ in the standard model structure of simplicial sets only when $N X$ is pointwise fibrant; that is, only in the rare situation when the vertices $X_{i}$ are groupoids. The goal of Theorem $\mathrm{B}^{I}$ is to find a condition on $X: I \rightarrow C a t$, weaker than assuming that $X$ is valued in groupoids, for which the nerve of $\operatorname{Hom}\left(I /{ }_{(-)}, X\right)$ is equivalent to the homotopy limit of $N X$.

Example 3.2 Let $I$ be the poset $\bullet \rightarrow \bullet \leftarrow \bullet$. A diagram indexed over this poset is a pullback diagram of categories $C \stackrel{f}{\rightarrow} D \stackrel{g}{\leftarrow} E$. There is an isomorphism of categories

$$
\operatorname{Hom}((\bullet \rightarrow \bullet \leftarrow \bullet) /(-), C \stackrel{f}{\rightarrow} D \stackrel{g}{\leftarrow} E) \cong f \downarrow g,
$$

where $f \downarrow g$ is the model for the homotopy pullback of Barwick and Kan [2]. The objects of $f \downarrow g$ are triples $(c, \gamma, e)$ consisting of objects $c \in C$ and $e \in E$, and a zig-zag of morphisms $\gamma=(f(c) \rightarrow d \leftarrow g(e))$ in the category $D$. This can also be described as the Grothendieck construction of the functor $f /{ }_{(-)} \times g /{ }_{(-)}: D \longrightarrow C a t$.

Let $i<I$ be the full subcategory of the under category $i / I$ of nonidentity maps with source $i$. Given a diagram $X: I \rightarrow$ Cat, we define $X_{i<:}(i<I) \rightarrow$ Cat to be the 
restriction of $X$ along the projection functor $i<I \rightarrow I$ that sends $i \rightarrow j$ to $j$. For every object $i$ of $I$, there is a functor

$$
m_{i}: X_{i} \longrightarrow \operatorname{Hom}\left((i<I) /(-), X_{i<}\right)
$$

that sends an object $x$ of $X_{i}$ to the natural transformation $m_{i}(x):(i<I) /(-) \rightarrow X_{i<}$ consisting of the constant functors $m_{i}(x)_{\alpha}:(i<I) / \alpha \rightarrow X_{j}$ that send every object to $\alpha_{*} x$. For the purpose of this paper, we say that a functor of small categories is a weak equivalence if its nerve is a weak equivalence of simplicial sets.

Definition 3.3 A diagram $X: I \rightarrow C$ at is Reedy quasifibrant if, for every object $i$ of $I$, the functor

$$
m_{i} \text { (-) }_{\text {Hom }}\left((i<I){ }_{(-)}, X_{i<}\right) \longrightarrow C a t
$$

sends every morphism in the category of natural transformations to a weak equivalence of categories.

This condition is reminiscent of the Reedy fibrancy conditions of diagrams indexed over a Reedy category. We explain the relationship between the two conditions more closely. The functor $m_{i}$ factors through the categorical limit

$$
m_{i}: X_{i} \longrightarrow \lim _{i \stackrel{\text { id }}{\longrightarrow} j} X_{j}=\operatorname{Hom}\left(*, X_{i<}\right) \longrightarrow \operatorname{Hom}\left((i<I) /(-), X_{i<}\right),
$$

where the second map is induced by the projection $(i<I) /{ }_{(-)} \rightarrow *$. The first functor is the $i$-matching functor of $X$. The nerves of the $m_{i}$ are thickenings of the matching maps of $N X$. The diagram $N X$ would be Reedy fibrant if the matching maps were Kan fibrations. If $X$ is Reedy quasifibrant then, by Quillen's Lemma [17, page 98] and Thomason's Theorem [20], the replacement of $m_{i}$ by the Grothendieck construction

$$
N X_{i} \simeq N\left(\operatorname{Hom} 2 m_{i} /{ }_{(-)}\right) \longrightarrow N \operatorname{Hom}\left((i<I) /(-), X_{i<}\right)
$$

is a quasifibration. In this sense, the condition of Theorem $\mathrm{B}^{I}$ is a Reedy quasifibrancy condition; hence the terminology.

Example 3.4 If we return to the diagram of categories $C \stackrel{f}{\rightarrow} D \stackrel{g}{\leftarrow} E$ of Example 3.2, we see that this is Reedy quasifibrant precisely when the functors $f /{ }_{(-)}, g /_{(-)}: D \rightarrow C a t$ send every morphism to a weak equivalence. 


\subsection{Finite homotopy limits of classifying spaces and the higher Quillen Theorem B}

Let $I$ be a small category, and suppose that the nerves of the under categories $N(i / I)$ are finite-dimensional simplicial sets for every object $i$ of $I$. We call a category $I$ with this property left-finite. Such a category has a canonical degree function $\mathrm{Ob} I \rightarrow \mathbb{N}$ that sends $i$ to the dimension of $N(i / I)$. This is the length of the longest sequence of nonidentity morphisms starting at $i$. The degree function induces a filtration

$$
I_{\leq 0} \subset I_{\leq 1} \subset \cdots \subset I,
$$

where $I_{\leq n}$ is the full subcategory of $I$ of objects of degree less than or equal to $n$. This filtration is finite precisely when $N I$ is itself finite-dimensional.

Let $X: I \rightarrow C a t$ be a diagram of categories. We choose the space

$$
\underset{I}{\operatorname{holim}} N X:=\operatorname{Hom}\left(N\left(I /\left(_{-}\right), F N X\right)\right.
$$

as a model for the homotopy limit of $N X$, where $N X \stackrel{\cong}{\rightarrow} F N X$ is a pointwise fibrant replacement of the diagram $N X$. The fibrant replacement induces a comparison map $N \operatorname{Hom}\left(I /{ }_{(-)}, X\right) \rightarrow \operatorname{holim}_{I} N X$.

Theorem $\mathbf{B}^{I}$ Let $I$ be a left-finite category, and let $X: I \rightarrow$ Cat be a Reedy quasifibrant diagram of categories as in Definition 3.3. There is a weak equivalence

$$
\operatorname{holim}_{n \in \mathbb{N}^{\circ p}} N \operatorname{Hom}\left(\left(I_{\leq n}\right) /(-), X_{\leq n}\right) \stackrel{\simeq}{\longrightarrow} \underset{I}{\operatorname{holim}} N X,
$$

where $X_{\leq n}$ is the restriction of $X$ to $I_{\leq n}$. In particular, if the nerve of $I$ is finitedimensional, the map

$$
N \operatorname{Hom}(I /(-), X) \stackrel{\simeq}{\rightarrow} \operatorname{holim} N X
$$

is a weak equivalence of simplicial sets.

Remark 3.5 Let us see how Theorem $\mathrm{B}^{I}$ relates to Quillen's Theorem B. Let us consider a diagram of categories $C \stackrel{f}{\rightarrow} D \stackrel{g}{\leftarrow} E$ on the poset $I=(\bullet \rightarrow \bullet \leftarrow \bullet)$. Theorem $\mathrm{B}^{I}$ tells us that the nerve of the category,

$$
\operatorname{Hom}\left((\bullet \rightarrow \bullet \leftarrow \bullet) /{ }_{(-)}, C \stackrel{f}{\rightarrow} D \stackrel{g}{\leftarrow} E\right) \cong f \downarrow g,
$$

is equivalent to the homotopy pullback of $N f$ and $N g$, provided that both functors $f /{ }_{(-)}, g /_{(-)}: D \rightarrow C a t$ send morphisms to equivalences (see Example 3.2 for the definition of $f \downarrow g)$. Barwick and Kan arrive at the same conclusion by assuming that just one among the functors $f /{ }_{(-)}$and $g /{ }_{(-)}$satisfies this property; see [2]. In 
particular, when $E=*$ is the trivial category, their result is equivalent to the original formulation of Quillen's Theorem B from [17]. The fact that one is able to weaken the quasifibrancy condition in the pullback case to only one of the functors $f /{ }_{(-)}$and $g /(-)$ is a special feature of squares. It is completely analogous to the fact that the pullback along a fibration is homotopy invariant, even though the pullback diagram itself is injectively fibrant only when both maps are fibrations; see eg [11, Proposition 13.3.9].

The proof of Theorem $\mathrm{B}^{I}$ is by induction on the filtration $I_{\leq 0} \subset I_{\leq 1} \subset \cdots \subset I$, by exploiting the fact that the complements $I_{n}=I_{\leq n} \backslash I_{\leq n-1}$ are discrete categories. The inductive step is based on a lemma that describes the interaction between natural transformations and Grothendieck constructions, which requires us to set up some notation. For any set of degree $n$ objects $U \subset I_{n}$, let $U \leq I$ be the union of the under categories $u / I$ for $u \in U$. Explicitly, its set of objects is

$$
\mathrm{Ob}(U \leq I)=\{(u \in U, \alpha: u \rightarrow i)\} .
$$

The set of morphisms $(u, \alpha) \rightarrow(v, \beta)$ is empty if $u$ and $v$ are different, and it is the set of morphisms $(u, \alpha) \rightarrow(u, \beta)$ in $u / I$ otherwise. Define $U<I$ to be the full subcategory of $U \leq I$ whose objects are nonidentity maps. Given a diagram of categories $X: I \rightarrow C a t$, we denote the corresponding restrictions by

$$
X_{U \leq}: U \leq I \rightarrow I \stackrel{X}{\rightarrow} \text { Cat }, \quad X_{U<:} U<I \rightarrow I \stackrel{X}{\rightarrow} \text { Cat },
$$

where $U \leq I \rightarrow I$ and $U<I \rightarrow I$ project onto the target. We recall from [20] that the Grothendieck construction of a functor $F: C \rightarrow C a t$ is the category $C \imath F$ with objects pairs $(c \in C, x \in \mathrm{Ob} F(c))$, and where a morphism $(c, x) \rightarrow(d, y)$ is a morphism $\alpha: c \rightarrow d$ in $C$ together with a morphism $\delta: \alpha_{*} x \rightarrow y$ in the category $F(d)$.

Lemma 3.6 Let $X: I \rightarrow$ Cat be a diagram of categories, and suppose that $I$ is left-finite. For every subset $U \subset I_{n}$, there is a natural isomorphism of categories

$$
\operatorname{Hom}\left((U \leq I) /(-), X_{U \leq}\right) \cong\left(\operatorname{Hom}\left((U<I) /{ }_{(-)}, X_{U<}\right)\left\langle F_{U}\right),\right.
$$

where $F_{U}: \operatorname{Hom}\left((U<I) /{ }_{(-)}, X_{U<}\right) \rightarrow$ Cat is the functor that sends a natural transformation $\Phi$ to the category

$$
F_{U}(\Phi)=\prod_{u \in U}\left(m_{u}\right) /\left(\left.\Phi\right|_{u<I}\right)
$$

Proof An object of the Grothendieck construction of Lemma 3.6 is a collection of functors $\left\{\Phi_{\alpha}:(U<I) / \alpha \rightarrow X_{i}\right\}_{\alpha}$ natural in the maps $\alpha: u \rightarrow i$ ranging over the 
objects of $U<i$, together with objects $x_{u} \in X_{u}$ for every $u \in U$, and compatible natural transformations for every $\alpha: u \rightarrow i$ :

$$
\gamma_{\alpha}: \alpha_{*} x_{u} \longrightarrow \Phi_{\alpha}
$$

Here $\alpha_{*} x_{u}:(U<I) / \alpha \rightarrow X_{i}$ is the constant functor with value $\alpha_{*} x_{u}$. Given such an object $(\Phi, \underline{x}, \underline{\gamma})$, define a natural transformation $\Psi:(U \leq I) /{ }_{(-)} \rightarrow X_{U \leq}$ as follows. An object of $(U \leq I) / \alpha$ is a factorization

$$
\underset{\searrow_{k}}{\stackrel{\alpha}{\longrightarrow}} i
$$

and an object of $(U<I) / \alpha$ is a similar factorization where the map $u \rightarrow k$ is not the identity. The functor $\Psi_{\alpha}:(U \leq I) / \alpha \rightarrow X_{i}$ is defined on objects by

$$
\Psi_{\alpha}\left(\begin{array}{c}
u \underset{k}{\stackrel{\alpha}{\longrightarrow}} i \\
\searrow_{k}
\end{array}\right)= \begin{cases}\alpha_{*} x_{u} & \text { if }(u \rightarrow k)=\mathrm{id}_{u}, \\
\Phi_{\alpha}\left(\begin{array}{c}
u \underset{k}{\searrow_{k}} i
\end{array}\right) & \text { if }(u \rightarrow k) \neq \mathrm{id}_{u} .\end{cases}
$$

The point here is that

$$
\Phi_{\alpha}\left(\begin{array}{c}
u \underset{k}{\stackrel{\alpha}{\longrightarrow}} i \\
\underset{k}{\searrow}
\end{array}\right)
$$

is defined precisely when $u \rightarrow k$ is not an identity. A morphism

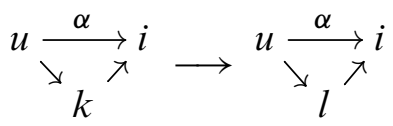

in $(U \leq I) / \alpha$ is a map $k \rightarrow l$ such that the two relevant triangles commute. Such a morphism is sent to

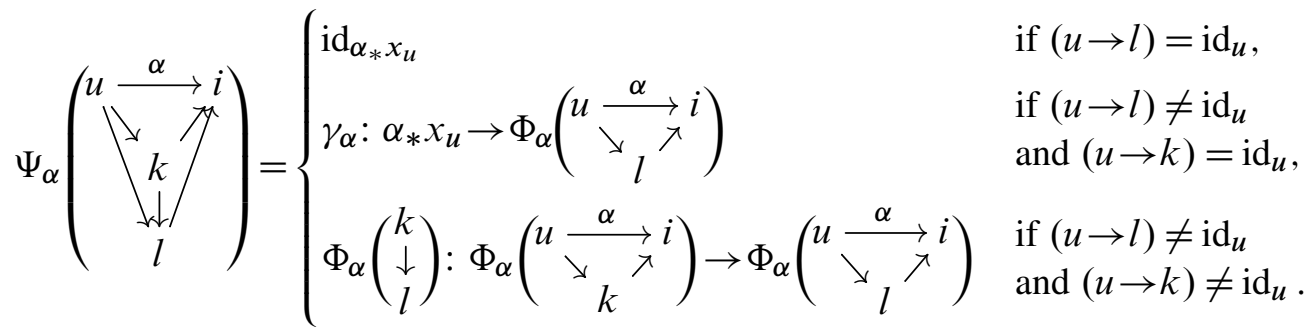

Notice that if $u \rightarrow l$ is the identity map on $u$, both $u \rightarrow k$ and $k \rightarrow l$ must be identities by degree considerations. This procedure defines a functor

$$
\left(\operatorname{Hom}\left((U<I){ }_{(-)}, X_{U<}\right) 2 F_{U}\right) \longrightarrow \operatorname{Hom}\left((U \leq I){ }_{(-)}, X_{U \leq}\right)
$$


on objects. We extend this on morphisms as follows. Unraveling the definitions of the Grothendieck construction and of the natural transformations category, we see that a morphism $(\Phi, \underline{x}, \underline{\gamma}) \rightarrow\left(\Phi^{\prime}, \underline{x}^{\prime}, \underline{\gamma}^{\prime}\right)$ in the left-hand category is a collection of compatible natural transformations $\lambda_{\alpha}: \Phi_{\alpha} \rightarrow \Phi_{\alpha}^{\prime}$ for every nonidentity map $\alpha: u \rightarrow i$ with $u \in U$, together with morphisms $f_{u}: x_{u} \rightarrow x_{u}^{\prime}$ in $X_{u}$ for every $u \in U$, which make the following squares commutative:

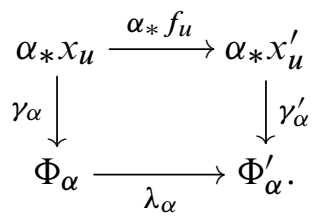

Such a pair $(\lambda, f)$ induces a morphism $\Psi \rightarrow \Psi^{\prime}$ between the associated natural transformations in $\operatorname{Hom}\left((U \leq I) /{ }_{(-)}, X_{U \leq}\right)$, defined at a nonidentity morphism $\alpha: u \rightarrow i$, by

$$
\Psi_{\alpha}=\Phi_{\alpha} \stackrel{\lambda_{\alpha}}{\longrightarrow} \Phi_{\alpha}^{\prime}=\Psi_{\alpha}^{\prime}
$$

and at an identity map $\operatorname{id}_{u}$ by $f_{u}: \Psi_{\mathrm{id}_{u}}=\alpha_{*} x_{u} \rightarrow \alpha_{*} x_{u}^{\prime}=\Psi_{\mathrm{id}_{u}}^{\prime}$. The resulting functor is an isomorphism of categories. Its inverse sends a natural transformation $\left\{\Psi_{\alpha}:(U \leq I) / \alpha \rightarrow X_{i}\right\}_{\alpha: u \rightarrow i}$ to the triple $(\Phi, \underline{x}, \underline{\gamma})$ consisting of the restrictions $\Phi_{\alpha}:(U<I) / \alpha \rightarrow(U \leq I) / \alpha \stackrel{\Psi_{\alpha}}{\longrightarrow} X_{i}$ for each $(\alpha: u \rightarrow i) \in U<I$, the objects $x_{u}=\left(\Psi_{u}: *=(U \leq I) / \mathrm{id}_{u} \rightarrow X_{u}\right)$, and natural transformations $\gamma_{\alpha}$ defined at an object

$$
\underset{k}{\stackrel{\alpha}{\longrightarrow}} i
$$

of $(U \leq I) / \alpha$ by the morphism in $X_{i}$ :

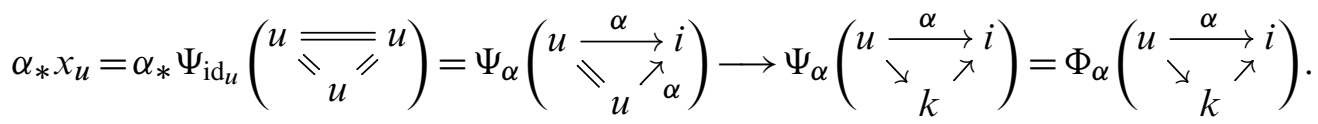

Here the second equality holds by naturality of $\Psi$, and the arrow is $\Psi_{\alpha}$ applied to the morphism

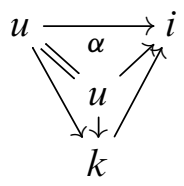


of $(U \leq I) / \alpha$ induced by the following factorization:

$$
u \underset{k}{\stackrel{\alpha}{\longrightarrow}} i
$$

The inverse can be extended similarly to morphisms.

Proof of Theorem $\mathbf{B}^{I}$ Let $N X \rightarrow F N X$ be a pointwise fibrant replacement of the diagram $N X$. We prove just below, by induction on $n$, that for every subset $U \subset I_{n}$, the map

$$
\text { (1) } N \operatorname{Hom}\left((U \leq I) /(-), X_{U \leq}\right) \cong \operatorname{Hom}\left(N(U \leq I) /(-), N X_{U \leq}\right) \stackrel{\simeq}{\rightarrow} \operatorname{holim}_{U \leq I} N X_{U \leq}
$$

is a weak equivalence. In particular, by choosing $U=I_{n}$, the category $I_{n} \leq I$ is $I_{\leq n}$, and we have an equivalence:

$$
N \operatorname{Hom}\left(\left(I_{\leq n}\right) /(-), X_{\leq n}\right) \stackrel{\simeq}{\rightarrow} \operatorname{holim}_{I_{\leq n}} N X_{\leq n} .
$$

If $N I$ is finite-dimensional, we have that $I=I_{\leq d}$ for some integer $d$, and the map $\left.N \operatorname{Hom}(I)_{(-)}, X\right) \rightarrow \operatorname{holim}_{I} N X$ is an equivalence. When $I$ is infinite, taking the homotopy limit over the maps induced by the filtration gives an equivalence:

$$
\operatorname{holim}_{n \in \mathbb{N} \text { op }} N \operatorname{Hom}\left(\left(I_{\leq n}\right) /(-), X_{\leq n}\right) \stackrel{\simeq}{\rightarrow} \operatorname{holim}_{n \in \mathbb{N} \text { op }} \underset{I_{\leq n}}{\operatorname{holim}} N X_{\leq n} .
$$

The structure maps holim $I_{\leq n} N X_{\leq n} \rightarrow$ holim $_{I_{\leq n-1}} N X_{\leq n-1}$ in the right-hand tower are Kan fibrations. Indeed, they are induced by mapping the cofibrations of diagrams of simplicial sets $\iota_{n} /_{(-)} \rightarrow I_{\leq n}$, where $\iota_{n}: I_{\leq n-1} \rightarrow I_{\leq n}$ is the inclusion, into the fibrant diagram $F N X_{\leq n}$. Hence the right-hand homotopy limit is equivalent to the categorical limit. Now each $\operatorname{Hom}\left(N\left(I_{\leq n}\right) /\left(_{-}\right), F N X_{\leq n}\right)$ is isomorphic to $\operatorname{Hom}\left(N j_{n} /\left(_{(-)}, F N X\right)\right.$, where $j_{n}: I_{\leq n} \rightarrow I$ is the inclusion. The right-hand limit is then

$$
\lim _{n \in \mathbb{N} \text { op }} \operatorname{Hom}\left(N\left(I_{\leq n}\right) /_{(-)}, F N X_{\leq n}\right) \cong \operatorname{Hom}\left(\operatorname{colim}_{n} N j_{n} /_{(-)}, F N X\right) \cong \underset{I}{\operatorname{holim}} N X .
$$

The last isomorphism holds as the category $j_{n} / i$ includes in $j_{n+1} / i$ for every object $i$ of $I$, with union $\bigcup_{n \in \mathbb{N}} j_{n} / i=I / i$. This will finish the proof of Theorem $\mathrm{B}^{I}$.

We are left with proving the inductive statement (1) above. The base induction step, $n=0$, relies on the fact that, for a subset $U \subset I_{0}$, the category $(U \leq I)$ is discrete with objects the identity maps $\operatorname{id}_{u}$ for $u \in U$. Therefore, the category $(U \leq I) / \operatorname{id}_{u}=\left\{\operatorname{id}_{u}\right\}$ is the one point category. It follows that $\operatorname{Hom}\left((U \leq I) /{ }_{(-)}, X_{U \leq}\right)$ is the product category

$$
\operatorname{Hom}\left((U \leq I){ }_{(-)}, X_{U \leq}\right)=\prod_{u \in U} X_{u},
$$


and the homotopy limit of $N X_{U \leq}$ is the product

$$
\operatorname{holim} N X_{U \leq}=\prod_{u \in U} F N X_{u}
$$

Since the product of simplicial sets preserves all equivalences (not only those between fibrant objects), the map

$$
N \prod_{u \in U} X_{u} \rightarrow \prod_{u \in U} F N X_{u}
$$

is an equivalence.

Now suppose that $N \operatorname{Hom}\left((U \leq I) /{ }_{(-)}, X_{U \leq}\right) \rightarrow$ holim $N X_{U \leq}$ is an equivalence for every subset $U \subset I_{n}$, and let $V$ be a subset of $I_{n+1}$. Let $\Phi:(V<I) /(-) \rightarrow X_{V<}$ be a natural transformation, and consider the commutative diagram:

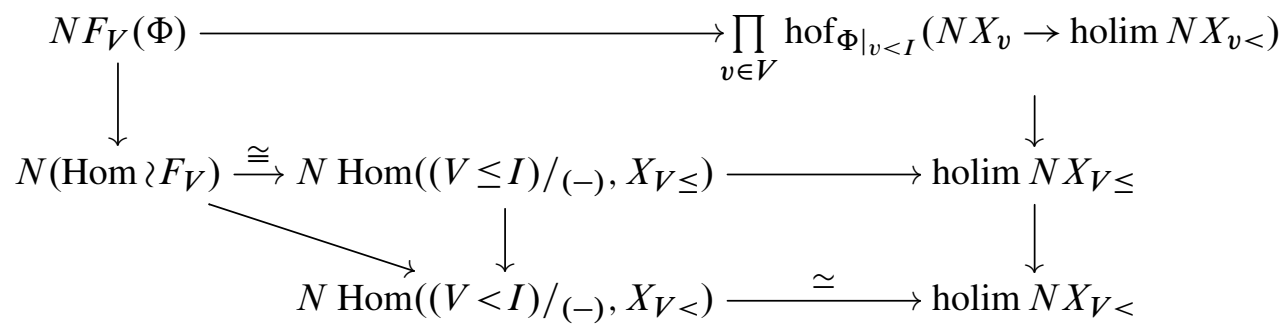

The bottom map is an equivalence by the inductive hypothesis, since $V<I=U \leq I$ for the subset of objects $U:=(V<I) \cap I_{n}$ of $I_{n}$. The isomorphism is from Lemma 3.6. By our assumption on the diagram $X$, the functor $F_{V}$ sends every morphism to a weak equivalence. Thus, by Quillen's Lemma [17, page 98] and Thomason's Theorem [20], the left-hand vertical sequence is a fiber sequence. Let us turn to the right-hand vertical sequence. Let $\iota: V<I \rightarrow V \leq I$ be the inclusion. The map induced by $\iota$ on homotopy limits is the restriction

$$
\underset{V \leq I}{\operatorname{holim}} N X_{V \leq} \longrightarrow \operatorname{Hom}\left(N \iota /(-), F N X_{V \leq}\right) \cong \underset{V<I}{\operatorname{holim}} N X_{V<}
$$

along the inclusion $\iota /{ }_{(-)} \rightarrow(V \leq I) /\left(_{(-)}\right.$. Since this is a cofibration of diagrams of simplicial sets and $F N X_{V \leq}$ is fibrant, the restriction map is a Kan fibration. Its point fiber over $\Phi$ is the product of total homotopy fibers

$$
\prod_{v \in V} \operatorname{hof}_{\left.\Phi\right|_{v<I}}\left(N X_{v} \rightarrow \operatorname{holim} N X_{v<}\right),
$$

and therefore the right-hand vertical sequence in the diagram above is also a fiber sequence. Thus, in order to finish our inductive proof, it is enough to show that the 
map on homotopy fibers

$$
\begin{aligned}
N F_{V}(\Phi)= & \prod_{v \in V} N\left(X_{v} \stackrel{m_{v}}{\longrightarrow} \operatorname{Hom}\left((v<I) /(-), X_{v<}\right)\right) /\left.\Phi\right|_{v<I} \\
& \prod_{v \in V} \operatorname{hof}_{\left.\Phi\right|_{v<I}}\left(N X_{v} \rightarrow \operatorname{holim} N X_{v<}\right)
\end{aligned}
$$

is an equivalence. The product components of this map factor as:

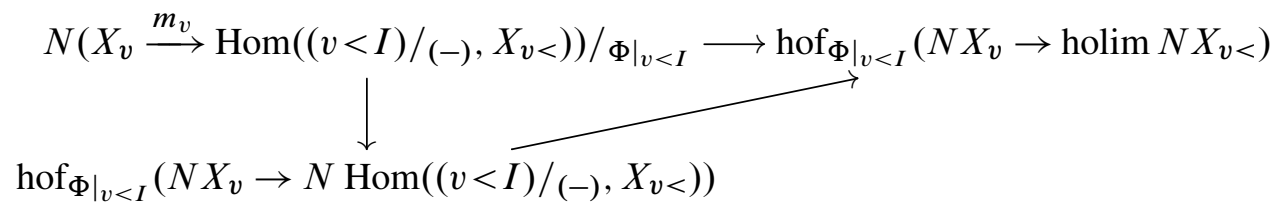

Our assumption on $X$ says that the functor $m_{v} /{ }_{(-)}$sends every map to a weak equivalence. Hence, by Quillen's Theorem B, the vertical map is an equivalence. The horizontal map is also an equivalence since, by the inductive hypothesis for the set $U:=(v<I) \cap I_{n}$, we have that the map $N \operatorname{Hom}\left((v<I) /(-), X_{v<}\right) \rightarrow \operatorname{holim} N X_{v<}$ is an equivalence.

The following result can be thought of as a generalization of Quillen's Theorem B to higher-dimensional cubes, with a higher Quillen Theorem A as a consequence.

Corollary 3.7 Let $n \geq 1$ be an integer, let $X: \mathcal{P}(n) \rightarrow$ Cat be a Reedy quasifibrant cube of categories, and let $\Phi: \mathcal{P}_{0}(-) \rightarrow X_{\varnothing<}$ be a natural transformation. The total homotopy fiber of $N X$ over $N \Phi$ is equivalent to the nerve of the over category $m_{\varnothing} / \Phi$. In particular, if the categories $m_{\varnothing} / \Phi$ are contractible, $N X$ is homotopy cartesian.

Proof Recall that the total homotopy fiber of $N X$ over $N \Phi$ is the homotopy fiber

$$
\operatorname{hof}_{\Phi}\left(N X_{\varnothing} \longrightarrow \operatorname{holim}_{\mathcal{P}_{0}(n)} N X_{\varnothing<)}\right)
$$

over the element in the homotopy limit defined by $N \Phi$. Clearly the restriction $X_{\varnothing<}$ of $X$ to $\mathcal{P}_{0}(n)$ is also Reedy quasifibrant, and by Theorem $\mathrm{B}^{I}$, the total homotopy fiber is equivalent to the homotopy fiber of

$$
\operatorname{hof}_{\Phi}\left(N X_{\varnothing} \stackrel{N m_{\varnothing}}{\longrightarrow} N \operatorname{Hom}\left(\mathcal{P}_{0}(-), X_{\varnothing<}\right)\right) \text {. }
$$

Since $m_{\varnothing} /_{(-)}$also sends all maps to equivalences, this is equivalent to $N m_{\varnothing} / \Phi$ by Quillen's Theorem B. 


\subsection{The equivariant Quillen Theorem B}

Let $G$ be a discrete group acting on a category $I$. We generalize the results of the previous section to $G$-homotopy limits of $G$-diagrams of categories $X: I \rightarrow C a t$. As in Section 3.2, it is going to be convenient to work with diagrams of simplicial sets instead of diagrams of topological spaces. The only difference is that one needs to perform the suitable fibrant replacements. We say that a morphism of $G$-diagrams of simplicial sets $f: Z \rightarrow Y$ is an equivalence if it is an equivalence of $G$-diagrams of topological spaces (as in Definition 1.4) after taking geometric realizations.

Definition 3.8 The $G$-homotopy limit of a $G$-diagram of simplicial sets $Y: I \rightarrow s$ Set is the $G$-simplicial set of natural transformations

$$
\underset{I}{\operatorname{holim}} Y=\operatorname{Hom}(N(I /(-)), F Y),
$$

where $F Y$ is a $G$-diagram of simplicial sets with an equivalence $Y \stackrel{\simeq}{\rightarrow} F Y$, with the property that $(F Y)_{i}$ is a fibrant $G_{i}$-simplicial set for every object $i$ in $I$.

It is proved in [7, Theorem 2.6] that such a replacement $Y \stackrel{\simeq}{\longrightarrow} F Y$ always exists, and that the $G$-homotopy limit functor preserves equivalences of $G$-diagrams of simplicial sets. As simplicial mapping spaces with fibrant target commute with geometric realizations, there is a $G$-equivalence $\left|\operatorname{holim}_{I} Y\right| \simeq \operatorname{holim}_{I}|Y|$ relating this construction with the $G$-homotopy limit of Section 1.1.

If $K, X: I \rightarrow C a t$ are two $G$-diagrams of categories, the category of natural transformations $\operatorname{Hom}(K, X)$ has an induced $G$-action by conjugation, and the isomorphism of simplicial sets $N \operatorname{Hom}(K, X) \cong \operatorname{Hom}(N K, N X)$ is $G$-equivariant. Composing this isomorphism with a fibrant replacement of $X$ leads to a $G$-equivariant map

$$
N \operatorname{Hom}(I /(-), X) \longrightarrow \underset{I}{\operatorname{holim}} N X \text {. }
$$

We extend the quasifibrancy condition of Definition 3.3 to the equivariant setting, and we show that this map is an equivalence. Suppose that $I$ is left-finite. The fixed-point categories $I^{H}$ are automatically left-finite for every subgroup $H$ of $G$. For every object $i$ of $I^{H}$, the under category $i / I$ has an action of $H$ that restricts to the subcategory $i<I$. The restriction of a $G$-diagram $X: I \rightarrow C a t$ to $i<I$ has a canonical structure of an $H$-diagram, and the functor $m_{i}: X_{i} \rightarrow \operatorname{Hom}\left((i<I) /(-), X_{i<}\right)$ of Definition 3.3 is $H$-equivariant. Let

$$
m_{i}^{H}: X_{i}^{H} \longrightarrow \operatorname{Hom}\left((i<I) /{ }_{(-)}, X_{i<}\right)^{H}
$$

be its restriction to the categories of fixed points. 
Definition 3.9 A $G$-diagram of categories $X: I \rightarrow C a t$ is $G$-Reedy quasifibrant if, for every subgroup $H$ of $G$ and every object $i$ of $I^{H}$, the functor $m_{i}^{H} /(-)$ sends every morphism to a weak equivalence of categories.

Theorem $\mathbf{B}_{\boldsymbol{G}}^{I}$ Let $I$ be a left-finite category with $G$-action, and let $X: I \rightarrow C a t$ be a $G$-diagram of categories. Suppose that $X$ is $G$-Reedy quasifibrant. Then there is a weak $G$-equivalence

$$
\operatorname{holim}_{n \in \mathbb{N}^{\text {op }}} N \operatorname{Hom}\left(\left(I_{\leq n}\right) /_{(-)}, X_{\leq n}\right) \stackrel{\simeq}{\longrightarrow} \operatorname{holim}_{I} N X .
$$

In particular, if the nerve of $I$ is finite-dimensional, the map $N \operatorname{Hom}\left(I /_{(-)}, X\right) \stackrel{\simeq}{\longrightarrow}$ holim $_{I} N X$ is a weak $G$-equivalence of simplicial $G$-sets.

Let $J$ be a finite $G$-set. The following result is our equivariant generalization of Quillen's Theorems A and B.

Corollary 3.10 Let $X: \mathcal{P}(J) \rightarrow$ Cat be a $G$-Reedy quasifibrant $J$-cube of categories. For every natural transformation $\Phi: \mathcal{P}_{0}(-) \rightarrow X_{\varnothing<}$, the nerve of the category $m_{\varnothing} / \Phi$ is $G_{\Phi}$-equivalent to the total homotopy fiber of the cube $N X$ over $N \Phi$. In particular, if all the categories $m_{\varnothing} / \Phi$ are $G_{\Phi}$-contractible, $B X$ is a homotopy cartesian $J$-cube of spaces.

Proof It is immediate from Theorem $\mathrm{B}_{G}^{I}$, using the argument of Corollary 3.7.

The proof of Theorem $\mathrm{B}_{G}^{I}$ is based on the same inductive argument in the proof of Theorem $\mathrm{B}^{I}$. The key ingredient for the induction step is an equivariant analogue of Lemma 3.6. If $Y: I \rightarrow C$ Cat is a $G$-diagram of categories, recall from Corollary 1.10 that its Grothendieck construction $I<Y$ has an induced $G$-action. Given a subset $U \subset I_{n}$, the $G$-action on $I$ induces a $G_{U}$-action on the categories $U \leq I$ and $U<I$, where $G_{U}$ is the subgroup of $G$ of elements that send $U$ to itself. The functor $F_{U}: \operatorname{Hom}\left((U<I) /(-), X_{U<}\right) \rightarrow$ Cat from Lemma 3.6, which sends $\Phi:(U<I) /(-) \rightarrow$ $X_{U<}$ to

$$
F_{U}(\Phi)=\prod_{u \in U}\left(m_{u}\right) /\left(\left.\Phi\right|_{u<I}\right)
$$

has a canonical $G_{U}$-structure. It is defined by conjugating the $G_{U}$-action on $U$ that indexes the product with the functors

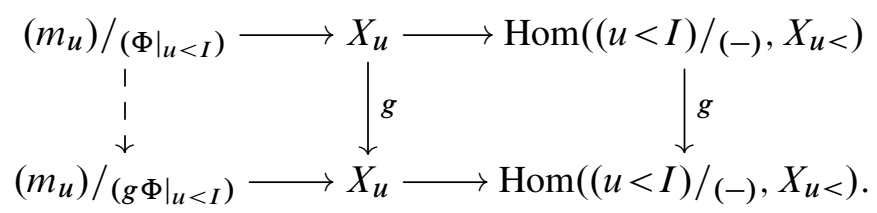


Hence the Grothendieck construction of $F_{U}$ inherits a $G_{U}$-action. The following lemma is an immediate consequence.

Lemma 3.11 For every subset $U \subset I_{n}$, the isomorphism of categories

$$
\operatorname{Hom}\left((U \leq I) /(-), X_{U \leq}\right) \cong\left(\operatorname{Hom}\left((U<I) /(-), X_{U<}\right)\left\{F_{U}\right)\right.
$$

of Lemma 3.6 is $G_{U}$-equivariant.

Proof of Theorem $\mathbf{B}_{\boldsymbol{G}}^{\boldsymbol{I}}$ For every group element $g$ of $G$, the automorphism $g$ of $I$ induces an isomorphism of categories $g: i / I \rightarrow g i / I$. It follows that the nerves $N(i / I)$ and $N(g i / I)$ have the same dimension, and that the degree function deg: $\mathrm{Ob} I \rightarrow \mathbb{N}$ is $G$-invariant. Hence the $G$-action restricts to the filtration

$$
I_{\leq 0} \subset I_{\leq 1} \subset \cdots \subset I_{\leq n} \subset \cdots \subset I,
$$

and the $G$-structure on $X: I \rightarrow$ Cat restricts to a $G$-structure on $X_{\leq n}: I_{\leq n} \rightarrow$ Cat . Let $N X \stackrel{\simeq}{\rightarrow} F N X$ be a pointwise fibrant replacement of $N X$, like in Definition 3.8. We prove by induction on $n$ that, for every subset $U \subset I_{n}$, the map

$$
N \operatorname{Hom}\left((U \leq I){ }_{(-)}, X_{U \leq}\right) \cong \operatorname{Hom}\left(N(U \leq I) /{ }_{(-)}, N X_{U \leq}\right) \longrightarrow \underset{U \leq I}{\operatorname{holim}}(F N X)_{U \leq}
$$

is a weak $G_{U}$-equivalence. Once this is established, the same argument in the proof of Theorem $\mathrm{B}^{I}$ finishes the proof of Theorem $\mathrm{B}_{G}^{I}$.

For $n=0$, the category $U \leq I$ is discrete, and the map above is the map of indexed products

$$
\prod_{u \in U} N X_{u} \longrightarrow \prod_{u \in U} F N X_{u}
$$

The fixed points of this map by a subgroup $H \leq G_{U}$ are isomorphic to the map

$$
\prod_{[u] \in U / H} N X_{u}^{H_{u}} \longrightarrow \prod_{[u] \in U / H} F N X_{u}^{H_{u}}
$$

for a choice of representatives in each $H$-orbit of $U$, where $H_{u}$ is the stabilizer group of $u$ in $H$. Each map $N X_{u}^{H_{u}} \rightarrow F N X_{u}^{H_{u}}$ is an equivalence of simplicial sets by assumption, and the map above is an equivalence.

Now suppose that the claim is true for $n$, and let $V$ be a subset of $I_{n+1}$. The sequence $N F_{V}(\Phi) \rightarrow N\left(\operatorname{Hom}\left\langle F_{V}\right) \cong N \operatorname{Hom}\left((V \leq I) /_{(-)}, X_{V \leq}\right) \rightarrow N \operatorname{Hom}\left((V<I) /_{(-)}, X_{V<}\right)\right.$ induced by the restriction map is a fiber sequence of simplicial $G_{V}-$ sets. This is because its restriction on fixed points of a subgroup $H \leq G_{V}$ is the sequence $N F_{V}(\Phi)^{H} \rightarrow N\left(\operatorname{Hom}\left\langle F_{V}\right)^{H} \cong N\left(\operatorname{Hom}^{H}\left\langle F_{V}^{H}\right) \rightarrow N \operatorname{Hom}\left((V<I) /(-), X_{V<}\right)^{H}\right.\right.$, 
where the functor $F_{V}^{H}: N \operatorname{Hom}\left((V<I){ }_{(-)}, X_{V<}\right)^{H} \rightarrow C a t$ sends an $H$-equivariant natural transformation $\Phi$ to

$$
F_{V}^{H}(\Phi)=\left(\prod_{v \in V}\left(m_{v}\right) /\left(\left.\Phi\right|_{v<I}\right)\right)^{H} \cong \prod_{[v] \in V / H} m_{v}^{H_{v}} /\left(\left.\Phi\right|_{v<I}\right) .
$$

By assumption, $m_{v}^{H_{v}} /{ }_{(-)}$sends every morphism to a weak equivalence, and thus so does $F_{V}^{H}$. It follows by the Lemma of [17, page 98] and [20] that $N F_{V}^{H}$ is indeed the homotopy fiber of the restriction map. The restriction map

$$
\text { holim } N X_{V \leq} \longrightarrow \operatorname{holim} N X_{V<}
$$

is a fibration of simplicial $G$-sets by an argument analogous to the one in the proof of Theorem $\mathrm{B}^{I}$. Its fiber is the product $\prod_{v \in V} \operatorname{hof}_{\left.\Phi\right|_{v<I}}\left(N X_{v} \rightarrow \operatorname{holim} N X_{v<}\right)$ of homotopy fibers. Therefore, it is sufficient to show that the map on homotopy fibers

$$
N F_{V}(\Phi) \longrightarrow \prod_{v \in V} \operatorname{hof}_{\left.\Phi\right|_{v<I}}\left(N X_{v} \rightarrow \operatorname{holim} N X_{v<}\right)
$$

is a $G_{V}$-equivalence. By taking fixed points, this is the case if, for all $v \in V$, the map

$$
N m_{v} /_{\left(\left.\Phi\right|_{v<I}\right)} \longrightarrow \operatorname{hof}_{\left.\Phi\right|_{v<I}}\left(N X_{v} \rightarrow \operatorname{holim} N X_{v<}\right)
$$

is a $G_{v}$-equivalence. This map factors as

$$
N m_{v} /\left(\Phi | _ { v < I } \longrightarrow \operatorname { h o f } _ { \Phi | _ { v < I } } \left(N X_{v} \rightarrow N \underset{\operatorname{hof}_{\left.\Phi\right|_{v<I}}}{\longrightarrow}\left(N X_{v} \stackrel{\downarrow}{\rightarrow} \operatorname{hom}\left((v<I) /(-), X_{v<}\right)\right)\right.\right.
$$

The top horizontal map is a $G_{v}$-equivalence since $m_{v}^{H} /(-)$ sends every morphism to a weak equivalence of categories for every subgroup $H$ of $G_{v}$. The vertical map is also a $G_{v}$ equivalence, as the map $N \operatorname{Hom}\left((v<I) /(-), X_{v<}\right) \rightarrow \operatorname{holim} N X_{v<}$ is a $G_{v}$-equivalence by the inductive hypothesis.

\section{Appendix: Connectivity of homotopy limits}

We prove a result about the connectivity of the space of natural transformations between two diagrams of spaces. This result was used in Corollary 1.14 to calculate the connectivity of the restriction map on $G$-homotopy limits. Let $G$ be a discrete group acting on a small category $I$. 
Proposition A.1 Let $K: I \rightarrow$ Top be a $G$-diagram of spaces, cofibrant in the model structure of [7, Theorem 2.6]. Suppose that, for every object $i$ of $I$, the simplicial set $N I / i$ is finite-dimensional, and that $K_{i}$ is a $G_{i}-C W$-complex. Then for every $G$-diagram of spaces $X: I \rightarrow$ Top, the $G$-fixed points of the space of natural transformations $\operatorname{Hom}(K, X)^{G}$ is either empty or $m$-connected, where

$$
m=\min _{i \in \mathrm{Ob} I} \min _{H \leq G_{i}}\left(\operatorname{Conn} X_{i}^{H}-\operatorname{dim} K_{i}^{H}\right),
$$

and where $G_{i}$ is the stabilizer group of the object $i$.

Corollary A.2 Let $X: I \rightarrow$ Top be a $G$-diagram of spaces and suppose that $N I / i$ is finite-dimensional for every object $i$ in $I$. Then $\left(\operatorname{holim}_{I} X\right)^{G}$ is either empty or $m$-connected, where

$$
m=\min _{i \in \mathrm{Ob} I} \min _{H \leq G_{i}}\left(\operatorname{Conn} X_{i}^{H}-\operatorname{dim} N I^{H} / i\right) .
$$

Remark A.3 For the trivial group $G=1$, this corollary shows that the homotopy limit of a diagram of spaces $X: I \rightarrow$ Top is

$$
\min _{i \in \operatorname{Ob} I}\left(\operatorname{Conn} X_{i}-\operatorname{dim} N I / i\right)
$$

connected (when nonempty). This result seems to be well known by the experts, but the author was not able to find a proof in the literature.

Proof of Corollary A.2 The $G$-diagram $N(I /-)$ is cofibrant in $\operatorname{Top}_{a}^{I}$ by [7, Example 2.19], and Proposition A.1 gives the formula of the statement.

Proof of Proposition A.1 For any pointed map $g: S^{k} \rightarrow \operatorname{Hom}(K, X)^{G}$ with $k$ smaller than the range of the statement, we need to build an extension of $g$ to the $(k+1)$-disc. By the adjunction between mapping spaces and products, this is the same as solving the extension problem

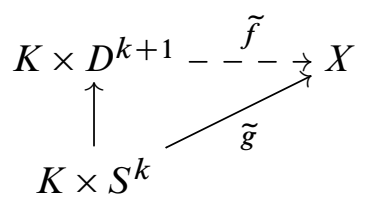

in the category of $G$-diagrams $\operatorname{Top}_{a}^{I}$. We define the extension $\tilde{f}$ by induction on a filtration of the objects of $I$ induced by the degree function deg: Ob $I \rightarrow \mathbb{N}$ defined by the dimensions of the over categories

$$
\operatorname{deg} i=\operatorname{dim} N I / i \text {. }
$$


This degree function is dual to the one used in Theorem $\mathrm{B}^{I}$. It is easy to verify that, for any nonidentity map $i \rightarrow j$, the inequality $\operatorname{deg}(i)<\operatorname{deg}(j)$ holds, and that the degree function is constant on $G$-orbits. This is an equivariant version of a directed Reedy category. For every positive integer $d$, define $I_{\leq d}$ to be the full subcategory of $I$ on objects of degree less than or equal to $d$, and $I_{d}$ the full subcategory of objects of degree $d$. Notice that the $G$-action restricts to these categories, and that $I_{d}$ is a discrete category.

For $i$ of degree -1 , the category $I_{\leq-1}$ is empty, and $\tilde{f}$ is the empty map. Now suppose that $\tilde{f}$ is defined as a natural transformation from the category $I_{\leq d-1}$. We start by defining $\tilde{f}_{i}$ on representatives of the orbits of the $G$-action on $I_{d}$. Let

$$
s: I_{d} / G \rightarrow I_{d}
$$

be a section for the quotient map, and let $z$ be an orbit in $I_{d} / G$. By degree reasons, the only morphisms of $I_{\leq d}$ involving $s(z)$ are maps $j \rightarrow s(z)$ with $j$ in $I_{\leq d-1}$. In order to be compatible with $I_{\leq d-1}$ and to extend $g$, the map $\widetilde{f}_{s(z)}$ needs to satisfy the following extension problem in Top:

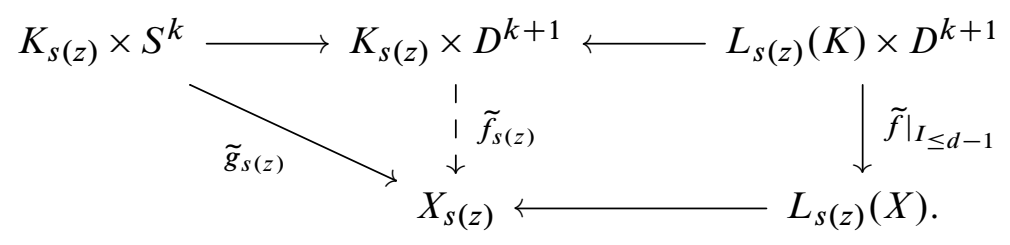

Here $L_{i}(Z)$ is the $i$-latching space of a $G$-diagram $Z \in \operatorname{Top}_{a}^{I}$ with vertices

$$
L_{i}(Z)=\underset{\substack{\neq \operatorname{fid}_{j} \\ j \rightarrow i}}{\operatorname{col}} Z_{j}
$$

The right-hand square expresses the compatibility of $\tilde{f}_{s(z)}$ with the extension already defined on $I_{\leq d-1}$. The stabilizer group $G_{S(z)}$ acts on all the spaces of the diagram, and both horizontal maps are cofibrations of $G_{i}$-spaces by cofibrancy of $K$. The extension problem above is equivalent to the extension problem of $G_{s(z)}$-spaces

$$
\begin{aligned}
L_{s(z)}(K) \times D^{k+1} U_{L_{s(z)}(K) \times S^{k}} K_{S(z)} \times S^{k} \longrightarrow X_{S(z)} & \downarrow_{K_{S(z)}} \times D^{k+1}
\end{aligned}
$$

and the vertical map is also a cofibration of $G_{i}$-spaces. The extension $\tilde{f}_{s(z)}$ can be defined inductively on the relative cells of the cofibration, provided that for any 
$G_{i} / H \times D^{n+1}-$ cell, the composition of $\tilde{g}_{s(z)}$ with the attaching map

$$
G_{i} / H \times S^{n} \rightarrow X_{i}
$$

is $G_{i}$-equivariantly null-homotopic. If $K_{i} \times D^{k+1}$ has a $G_{i} / H \times D^{n+1}$-cell, its fixed-points space $K_{i}^{H} \times D^{k+1}$ has an $(n+1)$-cell, and by dimension reasons, we must have

$$
n+1 \leq \operatorname{dim} K_{i}^{H} \times D^{k+1}=\operatorname{dim} K_{i}^{H}+k+1 \leq \operatorname{Conn} X_{i}^{H}+1 .
$$

The last inequality holds because $k$ is smaller than the range of the statement. Thus $\pi_{n} X_{i}^{H}$ is trivial, and any map $G_{i} / H \times S^{n} \rightarrow X_{i}$ is null-homotopic.

Now that $\tilde{f}_{s(z)}$ is defined on the representatives of the $G$-orbits of $I_{d}$, we extend it to the rest of $I_{d}$ by defining

$$
\tilde{f}_{i}: K_{i} \times D^{k+1} \stackrel{g^{-1}}{\longrightarrow} K_{S[i]} \times D^{k+1} \stackrel{\tilde{f}_{s[i]}}{\longrightarrow} X_{s[i]} \stackrel{g}{\longrightarrow} X_{i}
$$

for a choice of $g$ in $G$ such that $g s[i]=i$. The map $\tilde{f}_{i}$ does not depend on the choice of $g$ since $\tilde{f}_{s[i]}$ is $G_{i}$-equivariant. Moreover, the compatibility of $\widetilde{f}_{s[i]}$ with the maps $j \rightarrow s(z)$ insures that $\tilde{f}$ is natural on $I_{\leq d}$. That $\tilde{f}$ extends $\tilde{g}$, and that it is compatible with the $G$-structure, are easily verified.

Acknowledgments This project is a continuation of the work of [7], and part of a mission aimed at understanding a "genuine" context for equivariant homotopy theory. I wish to thank Kristian Moi for the many inspiring conversations we had over the last couple of years. I also want to thank Brian Munson and Ismar Volic for helpful correspondences, and Andrew Blumberg for his enduring support.

\section{References}

[1] J F Adams, Prerequisites (on equivariant stable homotopy) for Carlsson's lecture, from "Algebraic topology" (I Madsen, B Oliver, editors), Lecture Notes in Math. 1051, Springer, Berlin (1984) 483-532 MR764596

[2] C Barwick, D M Kan, Quillen Theorems $B_{n}$ for homotopy pullbacks of $(\infty, k)$ categories, preprint (2013) arXiv:1208.1777

[3] M Bökstedt, W C Hsiang, I Madsen, The cyclotomic trace and algebraic K-theory of spaces, Invent. Math. 111 (1993) 465-539 MR1202133

[4] A K Bousfield, D M Kan, Homotopy limits, completions and localizations, Lecture Notes in Mathematics 304, Springer, Berlin (1972) MR0365573 
[5] W Chachólski, J Scherer, Homotopy theory of diagrams, Mem. Amer. Math. Soc. 736, Amer. Math. Soc., Providence, RI (2002) MR1879153

[6] E Dotto, Equivariant calculus of functors and $\mathbb{Z} / 2$-analyticity of real algebraic $K$ theory, J. Inst. Math. Jussieu (2015) 1-55 published online

[7] E Dotto, K Moi, Homotopy theory of $G$-diagrams and equivariant excision, Alg. Geom. Topol. 2016 (2016) 325-395

[8] W G Dwyer, D M Kan, Function complexes for diagrams of simplicial sets, Nederl. Akad. Wetensch. Indag. Math. 45 (1983) 139-147 MR705421

[9] T G Goodwillie, Calculus, II: Analytic functors, K-Theory 5 (1991/92) 295-332 MR1162445

[10] T G Goodwillie, J R Klein, Multiple disjunction for spaces of Poincaré embeddings, J. Topol. 1 (2008) 761-803 MR2461855

[11] PS Hirschhorn, Model categories and their localizations, Mathematical Surveys and Monographs 99, Amer. Math. Soc. (2003) MR1944041

[12] S Jackowski, J Słomińska, G-functors, G-posets and homotopy decompositions of G-spaces, Fund. Math. 169 (2001) 249-287 MR1852128

[13] L G Lewis, Jr, Equivariant Eilenberg-Mac Lane spaces and the equivariant Seifertvan Kampen and suspension theorems, Topology Appl. 48 (1992) 25-61 MR1195124

[14] M G Lydakis, Homotopy limits of categories, J. Pure Appl. Algebra 97 (1994) 73-80 MR1310749

[15] M Merling, Equivariant algebraic $K$-theory, $\mathrm{PhD}$ thesis, The University of Chicago, Ann Arbor, MI (2014) MR3259967 Available at http://search.proquest.com/ docview/1559962143

[16] U Namboodiri, Equivariant vector fields on spheres, Trans. Amer. Math. Soc. 278 (1983) 431-460 MR701504

[17] D Quillen, Higher algebraic $K$-theory, I, from "Algebraic $K$-theory, I: Higher $K-$ theories (Proc. Conf., Battelle Memorial Inst.)" (H Bass, editor), Lecture Notes in Math. 341, Springer, Berlin (1973) 85-147 MR0338129

[18] S-i Takayasu, On stable summands of Thom spectra of $B(\mathbf{Z} / 2)^{n}$ associated to Steinberg modules, J. Math. Kyoto Univ. 39 (1999) 377-398 MR1709300

[19] J Thévenaz, P J Webb, Homotopy equivalence of posets with a group action, J. Combin. Theory Ser. A 56 (1991) 173-181 MR1092846

[20] R W Thomason, Homotopy colimits in the category of small categories, Math. Proc. Cambridge Philos. Soc. 85 (1979) 91-109 MR510404

[21] R Villarroel-Flores, The action by natural transformations of a group on a diagram of spaces, preprint (2004) arXiv:math/0411502 
[22] F Waldhausen, Algebraic K-theory of spaces, from "Algebraic and geometric topology" (A Ranicki, N Levitt, F Quinn, editors), Lecture Notes in Math. 1126, Springer, Berlin (1985) 318-419

Department of Mathematics, Massachusetts Institute of Technology 77 Massachusetts Avenue, Cambridge, MA 02139-4307, USA

dotto@mit.edu

Received: 19 June 2015 